\title{
Radiative damping of quiescent prominence oscillations
}

\author{
J. Terradas, R. Oliver, and J. L. Ballester
}

\author{
Departament de Física, Universitat de les Illes Balears, 07071 Palma de Mallorca, Spain \\ e-mail: ramon.oliver@uib.es; dfsjlbo@uib.es
}

Received 12 June 2001 / Accepted 14 August 2001

\begin{abstract}
Observations of quiescent prominence oscillations point out their finite lifetime, which suggests the presence of time damping. Recent analysis of ground-based observations of prominence oscillations (MolownyHoras et al. 1999) has revealed for the first time the temporal damping of velocity perturbations at different spatial locations within a quiescent prominence. Although the damping of wave motions can be explained using a variety of mechanisms, here we have adopted a very simple one, namely a radiative loss term based on Newton's law of cooling with constant relaxation time $\left(\tau_{R}\right)$, to analyse the influence of this type of radiative dissipation on the modes of oscillation of Kippenhahn-Schlüter and Menzel quiescent prominence models. Among other results, it is shown that slow modes are characterised by short damping times, which indicates that these oscillations are heavily damped, whereas fast modes are practically unaffected by this radiative dissipation and have very long damping times. Moreover, for a range of values of the radiative relaxation time the fundamental slow mode attains very large values of the period because of the destabilising effect of gravity. On the other hand, three-dimensional dispersion diagrams (i.e. plots of the real and imaginary parts of the frequency versus the wavenumber) are used to investigate the coupling between slow and fast modes. It turns out that far from adiabatic and isothermal conditions, the radiation mechanism can effectively decouple the two magnetoacoustic modes.
\end{abstract}

Key words. magnetohydrodynamics - Sun: oscillations - Sun: magnetic fields

\section{Introduction}

Seismology of solar coronal structures, such as prominences, serves to probe their nature and physical properties. During recent years, observational evidence about the presence of small-amplitude periodic oscillations or waves in quiescent prominences, seen at the solar limb or on the disk, has been gathered and is well summarised in reviews by Tsubaki (1988), Vršnak (1993) and Oliver (2000, 2001), to which the reader is referred for thorough information.

Up to now, observational evidence relies on the analysis of data provided by time series of several indicators such as Doppler velocity, line width, line intensity, etc. obtained with ground-based solar telescopes. These analysis have permitted to establish that these small amplitude oscillations are of local nature and to classify them in long-period oscillations ( $T>40 \mathrm{~min}$ ), intermediate-period $(10 \mathrm{~min}<T<40 \mathrm{~min})$ and short-period $(T<10 \mathrm{~min})$, although this classification does not reflect the origin or nature of the perturbations causing these different periods. On the other hand, so far there is little information about the wavelength, phase speed and lifetime of oscillations. Molowny-Horas et al. (1997) observed oscillations in different parts of a quiescent prominence and, for

Send offprint requests to: J. Terradas,

e-mail: jaume@hubble.uib.es one of these parts and assuming plane wave propagation, they were able to obtain lower limits to the group speed $\left(v_{\mathrm{g}} \geq 4.4 \mathrm{~km} \mathrm{~s}^{-1}\right)$, to the phase speed $\left(v_{\mathrm{p}} \geq 44 \mathrm{~km} \mathrm{~s}^{-1}\right)$ and to the wavelength $(\lambda \geq 20000 \mathrm{~km})$, and the lifetime of oscillations, which was around $12 \mathrm{~min}$. This last feature, the finite duration of oscillations, is an indication of their temporal growing and damping and constitutes an issue for which there has not been firm observational evidence until very recently.

Molowny-Horas et al. (1999) analysed time series of $\mathrm{H} \beta$ filtergrams of a prominence acquired at the VTT telescope of Sac Peak Observatory and showed that velocity perturbations with periods between 28 and 95 min exist at different spatial locations in the prominence and that the amplitude of oscillatory motions decreases in time (Fig. 1), with damping times between 101 and $377 \mathrm{~min}$. From this analysis, it also turns out that the damping per period (i.e. the ratio of the period to $2 \pi$ times the damping time) ranges from 0.03 to 0.11 .

Often, prominences have been represented by means of vertical plasma slabs having prominence physical properties and permeated by a horizontal magnetic field. The oscillations of these slab-like structures have been theoretically modeled using linear and adiabatic MHD waves (Joarder \& Roberts 1992, 1993a, 1993b; Oliver et al. 1992 (hereafter Paper I), 1993; Oliver \& Ballester 1995, 1996). A common approach is to represent the cool prominence 

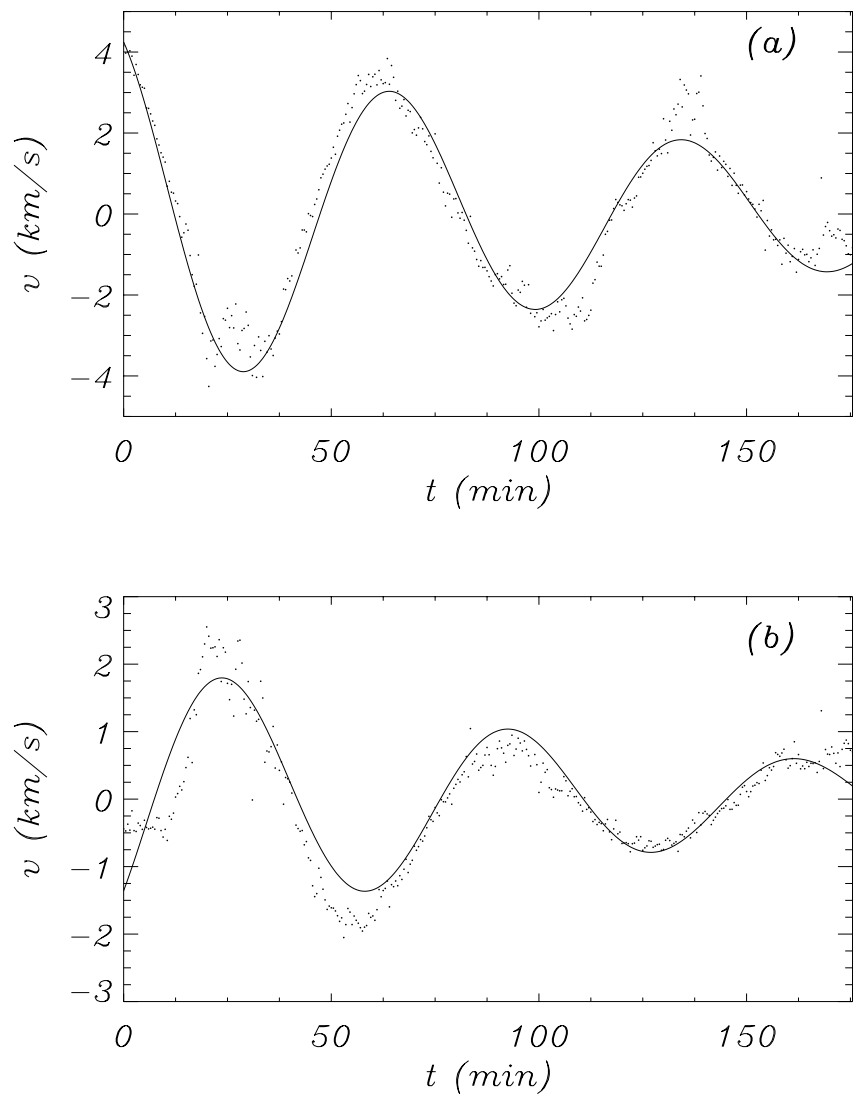

Fig. 1. Observed Doppler velocity (dots) and fitted function (continuous line) as functions of time at two different points in a quiescent prominence (Molowny-Horas et al. 1999). The period is $70 \mathrm{~min}$ in both points and the damping time is 140 and $101 \mathrm{~min}$, respectively. The function fitted to the observational data is of the form $v_{0} \cos (\omega t+\phi) \exp \left(-t / \tau_{\mathrm{D}}\right)$.

and the surrounding hot corona by means of two isothermal regions with a strong discontinuity in the temperature profile, while the magnetic field is assumed to be transverse or oblique to the configuration. In the case of transverse magnetic field and when the longitudinal wavenumber is not taken into account, fast and slow magnetoacoustic modes are decoupled from the Alfvén mode and, using appropriate boundary conditions, dispersion diagrams for the fast and slow modes can be obtained. These modes can be classified in internal, external or hybrid, depending on the zone responsible for their presence; in particular, hybrid modes are due to the joint presence of the prominence and the corona. Also, from the above mentioned diagrams the periods of oscillation can be obtained, which allows to identify the modes potentially responsible for long, intermediate- and short-period oscillations. In fact, short- and long-period oscillations in a configuration with transverse magnetic field are linked to internal and hybrid modes, respectively (Joarder \& Roberts 1992; Oliver et al. 1993). Other effects such as the influence of field-line curvature and gravity have also been investigated. Joarder \& Roberts (1993a) analysed the case of Menzel's prominence model subject to adiabatic perturbations and found that the period of the slow mode changes significantly when stratification is considered.

Although many different mechanisms, non-ideal effects, optically thin (or thick) radiation, retardation effects due to the finite time required for perturbations to travel between the prominence and photosphere (Schutgens 1997), wave leakage, etc., can be responsible for the damping of wave motions, the most simple approach is to take into consideration a radiative loss term in the energy equation based on Newton's law of cooling with a constant relaxation time $\left(\tau_{\mathrm{R}}\right)$. Then, the modes may have complex frequencies and so perturbations contain a term of the form $\exp \left(-t / \tau_{\mathrm{D}}\right)$, where $\tau_{\mathrm{D}}$ is the damping time. In essence, temperature fluctuations produced by the passage of a wave are assumed to be radiatively damped on a characteristic time scale $\tau_{\mathrm{R}}$. The limit $\tau_{\mathrm{R}} \rightarrow \infty$ corresponds to a perturbation that takes infinite time to damp, i.e. the adiabatic limit, while $\tau_{\mathrm{R}}=0$ corresponds to perturbations which are immediately damped, i.e. the isothermal limit. For $0<\tau_{\mathrm{R}}<\infty$ the situation is intermediate between the isothermal and the adiabatic limits and heat is exchanged between different locations at finite speed (Mihalas \& Mihalas 1984). In the case of a magnetised medium, this approach was used by Webb \& Roberts (1980) who analysed magnetohydrodynamic waves in an unbounded atmosphere in the presence of a uniform vertical magnetic field, by Bünte \& Bogdan (1994) who studied magnetoatmospheric waves in infinite and semi-infinite stratified, isothermal atmospheres, and by Banerjee et al. (1997) who have considered the effects of radiation losses on magnetoatmospheric-gravity waves in a stratified, magnetised atmosphere having a vertical magnetic field and bounded in the vertical direction.

In summary, our main goal in this paper is to investigate the influence of radiative dissipation, represented by means of Newton's law of cooling, on the normal modes of oscillation of two classical quiescent prominence models (the Kippenhahn-Schlüter and Menzel's solutions). Within this study, we find the slow and fast magnetoacoustic modes, having complex frequencies as a consequence of the damping mechanism, plus a radiation-induced or thermal mode, having a purely imaginary frequency and thus of no relevance to prominence oscillations. Then, we concentrate on the magnetoacoustic modes and investigate their dispersion diagrams, period of oscillations, damping times, ...

\section{Basic equations}

\subsection{Equilibrium configuration}

\subsubsection{Kippenhahn-Schlüter model}

In a Kippenhahn-Schlüter model, the prominence is considered as a thin isothermal sheet of cool plasma supported by magnetic tension against gravity and laterally compressed by magnetic pressure against the outward pressure gradient. We adopt a coordinate system with the two horizontal $x$ - and $y$-axes going across and 


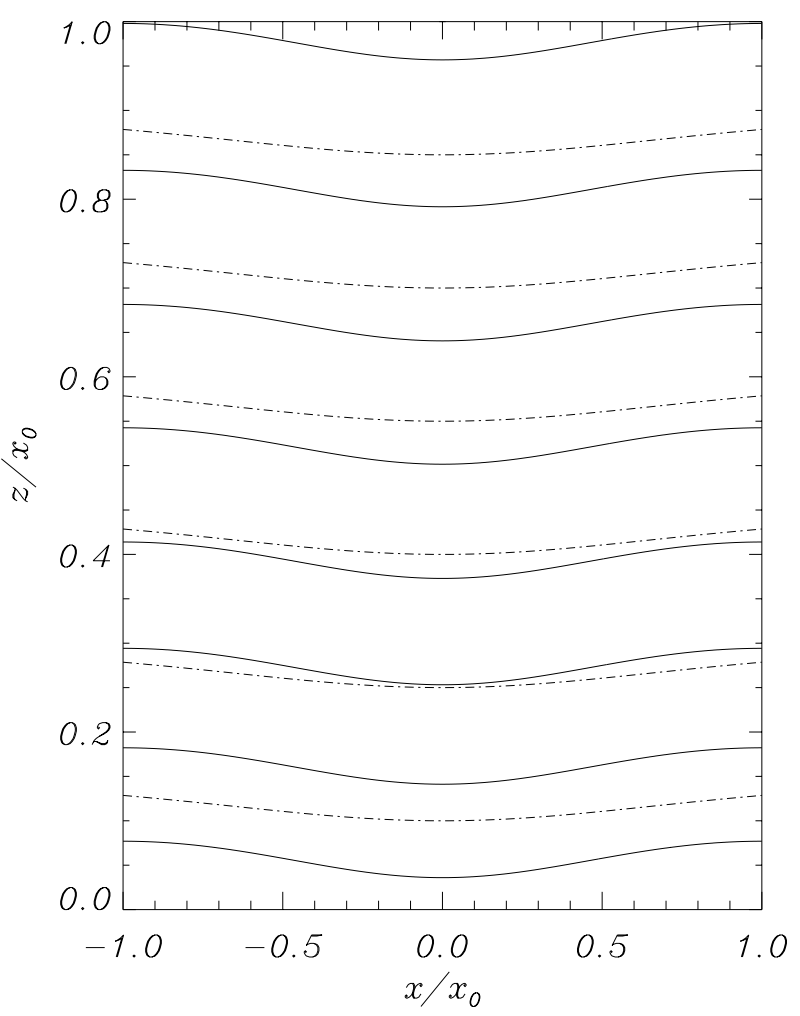

Fig. 2. Magnetic field lines in Menzel's prominence model (solid lines) and in the Kippenhahn-Schlüter model (dashed lines). The vertical stratification of Menzel's solution shows up in the separation between magnetic field lines, which grows with height. To compute these solutions the parameter values used are $B_{0 x}=5 \mathrm{G}, \rho_{0}(x=0, z=0)=1.2 \times 10^{-10} \mathrm{~kg} \mathrm{~m}^{-3}$, $T_{0}=7 \times 10^{3} \mathrm{~K}$ and $\tilde{\mu}=0.7$.

along the prominence, respectively, and the $z$-axis lying in the vertical direction. Then, the equilibrium variables are denoted as $\rho_{0}(x)$ for the density, $p_{0}(x)$ for the pressure and $\boldsymbol{B}_{0}\left(B_{0 x}, 0, B_{0 z}(x)\right)$ for the magnetic field, which gives place to a one-dimensional equilibrium since, although gravity is present in the model, there is no vertical stratification. Considering this configuration, the equilibrium equations are given by

$\frac{1}{\mu} B_{0 z} \frac{\mathrm{d} B_{0 z}}{\mathrm{~d} x}+\frac{\mathrm{d} p_{0}}{\mathrm{~d} x}=0$,

$\frac{1}{\mu} B_{0 x} \frac{\mathrm{d} B_{0 z}}{\mathrm{~d} x}=\rho_{0} g$,

$p_{0}=\frac{\rho_{0} R T_{0}}{\tilde{\mu}}$,

with $B_{0 x}=$ constant and $T_{0}=$ constant.

The boundary conditions used in integrating these equations are

$B_{0 z} \rightarrow \pm B_{z \infty}, \quad p_{0}=0 \quad$ as $x \rightarrow \pm \infty$

and $B_{0 z}=0$ at $x=0$ by symmetry. With these conditions, the equilibrium vertical magnetic field component and density are given by

$B_{0 z}(x)=B_{z \infty} \tanh \left(\frac{B_{z \infty}}{2 B_{0 x}} \frac{\tilde{\mu} g}{R T_{0}} x\right)$ and

$\rho_{0}(x)=\frac{B_{z \infty}^{2}}{2 \mu} \frac{\tilde{\mu}}{R T_{0}} \operatorname{sech}^{2}\left(\frac{B_{z \infty}}{2 B_{0 x}} \frac{\tilde{\mu} g}{R T_{0}} x\right)$,

with the pressure determined from Eqs. (6) and (3). The shape of magnetic field lines in a Kippenhahn-Schlüter model with typical prominence temperature and magnetic field strength can be seen in Fig. 2.

\subsubsection{Menzel's model}

This is a two-dimensional, isothermal prominence model in which pressure has the separable form

$p_{0}(x, z)=P(x) \exp \left(-\frac{z}{H_{\mathrm{B}}}\right)$

where $H_{\mathrm{B}}$ is the magnetically-modified scale height. In addition, the magnetic field is

$\boldsymbol{B}_{0}(x, z)=\left(-\frac{\partial F}{\partial z}, 0, \frac{\partial F}{\partial x}\right)$

with the flux function, $F$, given by

$F(x, z)=f(x) \exp \left(-\frac{z}{2 H_{\mathrm{B}}}\right)$.

After imposing force balance, $f(x)$ must satisfy the differential equation

$4 H_{\mathrm{B}}^{2} \frac{\mathrm{d}^{2} f}{\mathrm{~d} x^{2}}=-f+q \beta_{0} f_{0}\left(\frac{f_{0}}{f}\right)^{(2 q+1)}$,

with $q=H_{\mathrm{B}} / H_{0}-1, H_{0}=R T_{0} / \tilde{\mu} g$ being the hydrostatic scale height, and $\beta_{0}$ the plasma $\beta$ at $x=z=0$.

Equation (10) can be solved numerically with the boundary conditions

$f(x)=f_{0}, \frac{\mathrm{d} f}{\mathrm{~d} x}=0 \quad$ at $x=0$.

Then, the flux function can be determined from Eq. (9) and the magnetic field follows from Eq. (8), while the plasma pressure is

$p_{0}(x, z)=\frac{1}{2 \mu} \frac{\beta_{0}}{4 H_{\mathrm{B}}^{2}} f_{0}^{2}\left[\frac{f_{0}}{f(x)}\right]^{2 q} \exp \left(-\frac{z}{H_{\mathrm{B}}}\right)$.

The density can now be computed from this expression and the ideal gas law.

For $q>1$ and $q \beta_{0}>1$ the function $f(x)$ has a minimum, and thus the pressure has a maximum, at $x=0$, resulting in a prominence-like equilibrium in which the tension of concave magnetic field lines can support the dense material (see Fig. 2). Taking a typical value of the plasma $\beta$ in the central part of the prominence, $\beta_{0}=0.1$, the above constraints reduce to $q>10$. Now, since Menzel's solution is periodic in the $x$-direction, it is usual to consider the prominence confined between the first maxima of $f(x)$ on both sides of the origin. Given this "definition" of prominence width, the larger the parameter $q$, the thicker the prominence, so in practice there is 
also an upper bound for allowed values of $q$. We here select $q=14$, for which the prominence edge is at $x_{0}=5236 \mathrm{~km}$ from the prominence centre, with $2 x_{0}$ the prominence width. To investigate the effect of the magnetic field line curvature we have also selected $q=18$ with the same prominence width.

In the limit $q \beta_{0}=1$ Menzel's model reduces to a purely horizontal, stratified magnetic field. In this case, the magnetically modified scale height is given by

$H_{\mathrm{B}}=\frac{p_{0}+B_{0}^{2} / 2 \mu}{\rho_{0} g}$.

\subsection{Linearised equations}

To study the linear oscillations of the equilibria described in the previous section we use the standard set of linearised MHD equations,

$\frac{\partial \rho_{1}}{\partial t}=-\rho_{0} \nabla \cdot \boldsymbol{v}-\boldsymbol{v} \cdot \nabla \rho_{0}$

$\rho_{0} \frac{\partial \boldsymbol{v}}{\partial t}=-\nabla p_{1}-\nabla p_{1 \mathrm{~m}}+\mathcal{T}_{1}-\rho_{1} g \hat{e}_{z}$,

$\frac{\partial \boldsymbol{B}_{1}}{\partial t}=\nabla \times\left(\boldsymbol{v} \times \boldsymbol{B}_{0}\right)$

$\frac{p_{1}}{p_{0}}=\frac{\rho_{1}}{\rho_{0}}+\frac{T_{1}}{T_{0}}$

where the subscripts " 0 " and " 1 " denote equilibrium and perturbed magnitudes, and the perturbation to the magnetic tension $\mathcal{T}_{1}$ and to the magnetic pressure $p_{1 \mathrm{~m}}$ are defined as

$\mathcal{T}_{1}=\frac{1}{\mu}\left[\left(\boldsymbol{B}_{0} \cdot \nabla\right) \boldsymbol{B}_{1}+\left(\boldsymbol{B}_{1} \cdot \nabla\right) \boldsymbol{B}_{0}\right]$,

$p_{1 \mathrm{~m}}=\frac{\boldsymbol{B}_{0} \cdot \boldsymbol{B}_{1}}{\mu}$.

The above equations are supplemented with the linearised energy equation, which is written as

$\frac{1}{T_{0}} \frac{\partial T_{1}}{\partial t}+(\gamma-1) \nabla \cdot \boldsymbol{v}=-\frac{1}{\tau_{\mathrm{R}}} \frac{T_{1}}{T_{0}}$

where radiative losses have been represented by means of "Newton's law of cooling", $\tau_{\mathrm{R}}$ being the so-called radiative relaxation time. In the incompressible regime, this parameter determines the characteristic time it takes for the temperature to return to its equilibrium value after the system has been perturbed. Hence, the limit $\tau_{\mathrm{R}} \rightarrow \infty$ corresponds to disturbances that are never damped because there is no heat transfer between plasma elements, i.e. adiabatic disturbances, while the limit $\tau_{\mathrm{R}}=0$ corresponds to waves that enormously favour heat exchange and which maintain the plasma isothermal. Finite, non-zero values of $\tau_{\mathrm{R}}$ result in intermediate situations.

Perturbations are assumed to be of the form $f_{1}(\boldsymbol{r}, t)=$ $f_{1}(\boldsymbol{r}) \exp (i \omega t), \omega$ being the frequency. All one has to do now is to insert perturbations into Eqs. (14)-(16) and (20) to derive the expressions governing the linear MHD oscillations of the system. Nevertheless, the process of deriving the wave equations for an isothermal equilibrium, such as the Kippenhahn-Schlüter or Menzel's ones, can be simplified by following Bünte \& Bogdan (1994). These authors pointed out that, in an isothermal atmosphere, the effect of Newton's cooling can be easily taken into account by substituting in the adiabatic wave equations the ratio of specific heats, $\gamma$, by the complex $\hat{\gamma}$ defined as

$\hat{\gamma}=\frac{1+i \omega \tau_{\mathrm{R}} \gamma}{1+i \omega \tau_{\mathrm{R}}}$

Hence, we only have to replace in the adiabatic wave equations the adiabatic sound speed, $c_{\mathrm{s}}=\left(\gamma p_{0} / \rho_{0}\right)^{1 / 2}$, by the complex $\hat{c}_{\mathrm{s}}$ given by

$\hat{c}_{\mathrm{s}}^{2}=\hat{\gamma} \frac{p_{0}}{\rho_{0}}$

Note that in the adiabatic limit $\hat{\gamma}=\gamma$ while in the isothermal limit $\hat{\gamma}=1$, which means that the sound speed in the isothermal case is lower than in the adiabatic situation.

Before writing down the wave equations a last remark about the vertical dependence of perturbations is in order. Physical variables in the Kippenhahn-Schlüter model only depend on $x$, so that the spatial part of perturbations can be written as $f_{1}(\boldsymbol{r})=f_{1}(x) \exp \left(-i k_{z} z\right)$, where propagation in the $y$-direction is discarded. In Menzel's model, the physical variables have an exponential height dependence, but it is still possible to express the spatial part of disturbances as $f_{1}(\boldsymbol{r})=f_{1}(x) \exp \left[\left(-i k_{z}+1 / 2 H_{\mathrm{B}}\right) z\right]$. Hereafter we write the derivatives in the $z$-direction as $-i k_{z}$, keeping in mind that when Menzel's model is considered, $k_{z}$ must be replaced by $k_{z}+i / 2 H_{\mathrm{B}}$.

Under the previous assumptions, the linearised continuity equation, the perturbed gas law, the energy equation and the $x$ - and $z$-components of the induction equation reduce to

$i \omega \rho_{1}=-\rho_{0} \frac{\mathrm{d} v_{x}}{\mathrm{~d} x}+i k_{z} \rho_{0} v_{z}-\frac{\partial \rho_{0}}{\partial x} v_{x}-\frac{\partial \rho_{0}}{\partial z} v_{z}$,

$i \omega p_{1}=-\hat{\gamma} p_{0} \frac{\mathrm{d} v_{x}}{\mathrm{~d} x}+i k_{z} \hat{\gamma} p_{0} v_{z}-\frac{\partial p_{0}}{\partial x} v_{x}-\frac{\partial p_{0}}{\partial z} v_{z}$

$\left(i \omega+\frac{1}{\tau_{\mathrm{R}}}\right) T_{1}=(\gamma-1) T_{0}\left(-\frac{\mathrm{d} v_{x}}{\mathrm{~d} x}+i k_{z} v_{z}\right)$,

$i \omega B_{1 x}=-i k_{z} B_{0 z} v_{x}+i k_{z} B_{0 x} v_{z}+\frac{\partial B_{0 z}}{\partial z} v_{x}-\frac{\partial B_{0 x}}{\partial z} v_{x}$

and

$i \omega B_{1 z}=-B_{0 z} \frac{\mathrm{d} v_{x}}{\mathrm{~d} x}+B_{0 x} \frac{\mathrm{d} v_{z}}{\mathrm{~d} x}-\frac{\partial B_{0 z}}{\partial x} v_{x}+\frac{\partial B_{0 x}}{\partial x} v_{z}$.

We next use Eqs. (26) and (27) to calculate the perturbed Lorentz force and then insert $\rho_{1}$ and $p_{1}$ from Eqs. (23) and (24) into Eq. (15), whose $x$ - and $z$-components reduce to

$\frac{\mathrm{d}^{2} v_{x}}{\mathrm{~d} x^{2}}=q_{1} \frac{\mathrm{d} v_{x}}{\mathrm{~d} x}+q_{2} \frac{\mathrm{d} v_{z}}{\mathrm{~d} x}+q_{3} v_{x}+q_{4} v_{z}$ 

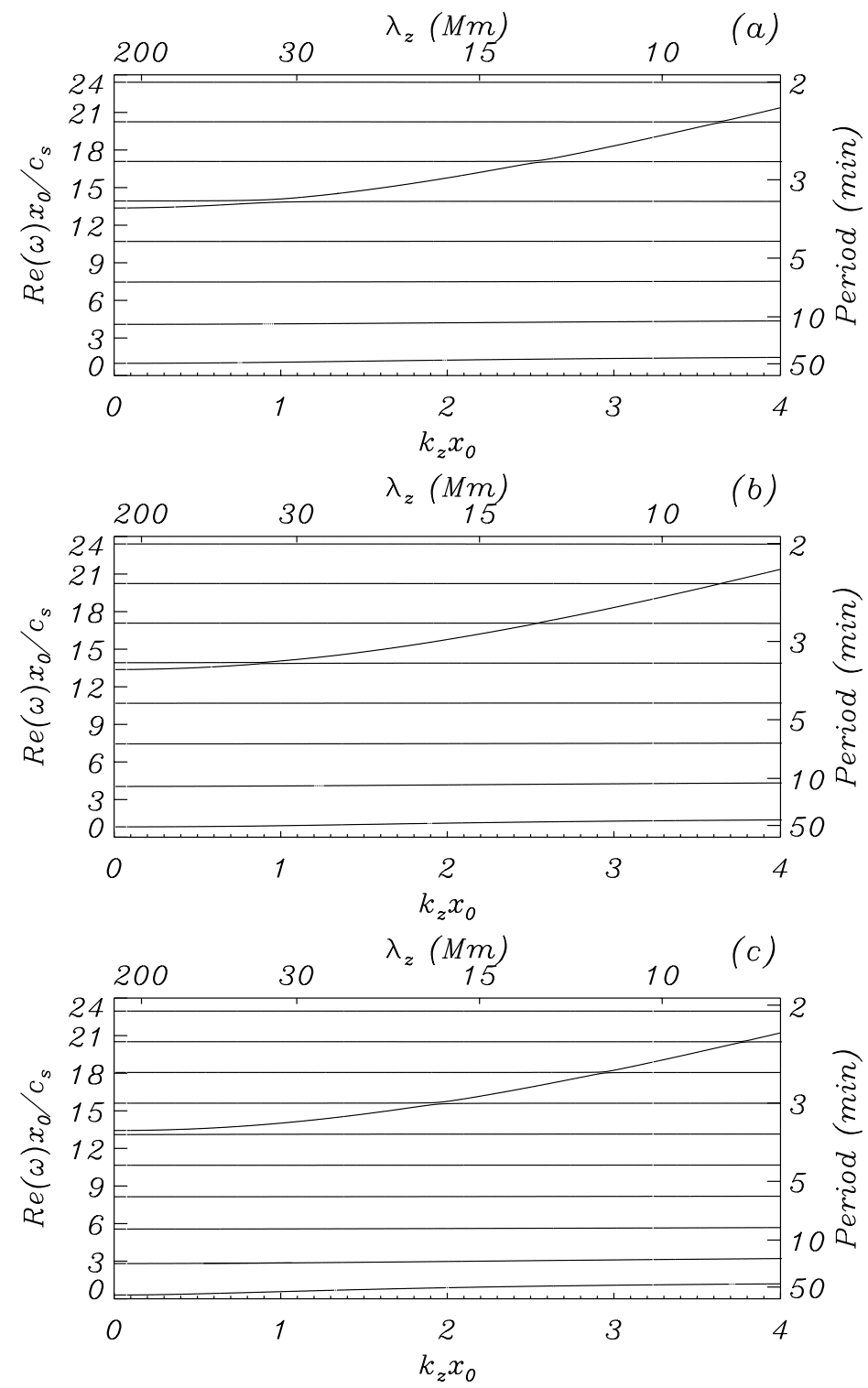

Fig. 3. Dispersion diagrams of slow and fast magnetoacoustic modes in Menzel's equilibrium with a) $\tau_{\mathrm{R}} c_{\mathrm{s}} / x_{0} \rightarrow \infty$ (adiabatic perturbations), b) $\tau_{\mathrm{R}} c_{\mathrm{s}} / x_{0}=1$ (radiatively damped perturbations) and $\left.\mathbf{c}\right) \tau_{\mathrm{R}}=0$ (isothermal perturbations). The modes in the diagram correspond to solutions with $v_{x}$ even and $v_{z}$ odd about $x=0$.

and

$\frac{\mathrm{d}^{2} v_{z}}{\mathrm{~d} x^{2}}=q_{5} \frac{\mathrm{d} v_{x}}{\mathrm{~d} x}+q_{6} \frac{\mathrm{d} v_{z}}{\mathrm{~d} x}+q_{7} v_{x}+q_{8} v_{z}$.

Expressions for the complex coefficients $q_{1}-q_{8}$ are given in Appendix A.

Equations (28) and (29) govern the propagation of fast and slow modes and constitute the main result of this section. The main difference between these equations and those solved in previous adiabatic studies bears in both the complex sound speed, $\hat{c}_{\mathrm{s}}$, and complex frequency, $\omega$.

We supplement the above differential equations with the rigid boundary conditions

$v_{x}\left( \pm x_{0}\right)=v_{z}\left( \pm x_{0}\right)=0$,

where $x_{0}$ is the half-width of the prominence.
On the other hand, the expressions that describe Alfvén waves are obtained from the $y$-component of Eqs. (15) and (16), which couple together $v_{y}$ and $B_{y}$, and will be not considered here.

\section{Results}

The coupled system of ordinary differential Eqs. (28) and (29), subject to the boundary conditions (30), has been solved following the procedure outlined in Paper I. For both the Kippenhahn-Schlüter and Menzel's models, we have considered the following parameter values: $B_{0 x}=5 \mathrm{G}, \rho_{0}(x=0, z=0)=1.2 \times 10^{-10} \mathrm{~kg} \mathrm{~m}^{-3}, T_{0}=$ $7 \times 10^{3} \mathrm{~K}$ and $\tilde{\mu}=0.7$, for which $c_{\mathrm{s}}=11.76 \mathrm{~km} \mathrm{~s}^{-1}$ and the plasma $\beta$ attains the maximum value 0.1 at the prominence centre. Furthermore, the prominence half-width $x_{0}$ has been taken equal to $5236 \mathrm{~km}$ in all calculations, which 


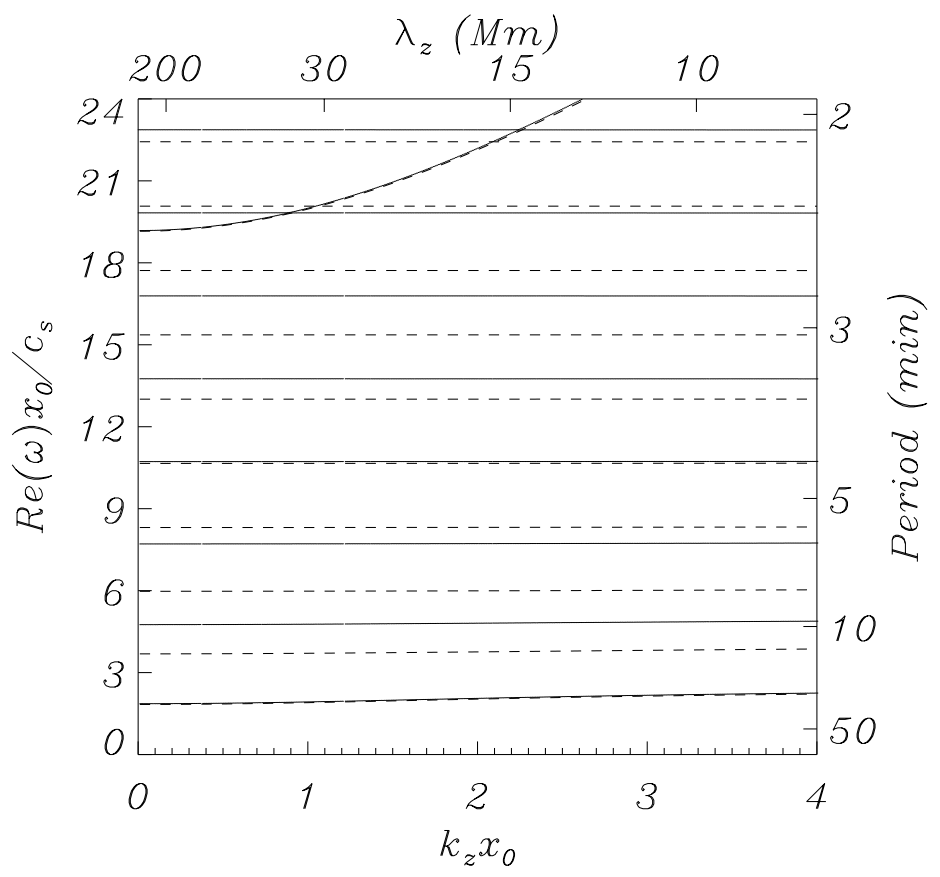

Fig. 4. Dispersion diagram of slow and fast magnetoacoustic modes in a Kippenhahn-Schlüter equilibrium with $\tau_{\mathrm{R}} c_{\mathrm{s}} / x_{0} \rightarrow \infty$, i.e. adiabatic perturbations (solid lines), and $\tau_{\mathrm{R}}=0$, i.e. isothermal perturbations (dashed lines). The modes in the diagram correspond to solutions with $v_{x}$ even and $v_{z}$ odd about $x=0$.

corresponds to the position of maximum height of magnetic field lines in Menzel's model with $q=14$.

\subsection{Dispersion diagrams}

To study the effect of Newton's radiative cooling on the magnetoacoustic modes of both equilibria, we have first plotted the dispersion diagrams for different values of $\tau_{R}$ (Figs. 3 and 4). We concentrate in oscillatory modes with $v_{x}$ even and $v_{z}$ odd about $x=0$, although the conclusions presented here are valid for modes having the opposite parity. Three representative values of $\tau_{\mathrm{R}}$ have been considered, namely $\tau_{\mathrm{R}} \rightarrow \infty$ corresponding to the adiabatic case (Figs. 3a and 4$) ; \tau_{\mathrm{R}}=445 \mathrm{~s}\left(\tau_{\mathrm{R}} c_{\mathrm{s}} / x_{0}=1\right)$ corresponding to an intermediate case (Fig. $3 \mathrm{~b}$ ); and $\tau_{\mathrm{R}}=0$ which corresponds to the isothermal case (Figs. 3c and 4). Here, it is worth to point out that the value of $\tau_{\mathrm{R}}$ in solar prominences is not very well known. Assuming optically thin radiation, Hood (1992) estimated a value for the radiative time around $1 \mathrm{~s}$ in prominences and $3000 \mathrm{~s}$ in the corona. The assumption of optically thin radiation can be considered valid in the prominence-corona transition region (PCTR), where the radiative times goes from $10 \mathrm{~s}$, at the prominence boundary, to $3000 \mathrm{~s}$, at the corona boundary. Although the above values of $\tau_{R}$ in the corona and PCTR seem quite reliable, there is a much greater uncertainty about the correct value of this parameter in prominences, which may well be quite different from $1 \mathrm{~s}$ (i.e. $\tau_{\mathrm{R}} c_{\mathrm{S}} / x_{0}=2.2 \times 10^{-3}$ ).

Such as expected, it turns out that for both equilibrium solutions, the fast mode frequency appears practically unaffected by the radiation mechanism (Roberts 2000), while slow mode frequencies become appreciably smaller when going from the adiabatic to the isothermal limit, due to the decrease of the effective value of the sound speed. In addition, it must be pointed out that in the case of Menzel's prominence model (Fig. 3), the fundamental slow mode shows a dispersive behaviour, since its frequency changes with the vertical wavenumber, while slow mode harmonics are non dispersive. It is interesting to note that the fundamental slow mode in Menzel's equilibrium achieves rather long periods (up to $154 \mathrm{~min}$ ) for very small vertical wavenumbers and $\tau_{\mathrm{R}}=0$ (see Fig. $3 \mathrm{c}$ ).

\subsection{Influence of $\tau_{\mathrm{R}}$ on mode properties}

We next concentrate on the fundamental slow and fast modes in Menzel's prominence model and plot some parameters versus the radiative relaxation time. These parameters are the real part of the frequency, representative of the period of oscillation, the inverse of the imaginary part of the frequency (equal to the damping time, $\tau_{\mathrm{D}}=$ $1 / \operatorname{Im}(\omega))$ and the damping per period (introduced by Stein \& Spiegel 1967), given by the ratio of the imaginary to the real part of the frequency $\left(D_{\mathrm{p}}=\operatorname{Im}(\omega) / \operatorname{Re}(\omega)\right)$. For the fundamental slow mode, Figs. $5 \mathrm{a}$ and $5 \mathrm{~b}$ show that the frequency transition from the isothermal to the adiabatic situation takes place in the range of cooling times $4.45-4450 \mathrm{~s}\left(10^{-2}-10^{1}\right.$ in dimensionless units), while almost simultaneously the damping time goes through a minimum. Moving away from this minimum, either to the adiabatic or to the isothermal limits, the damping time 


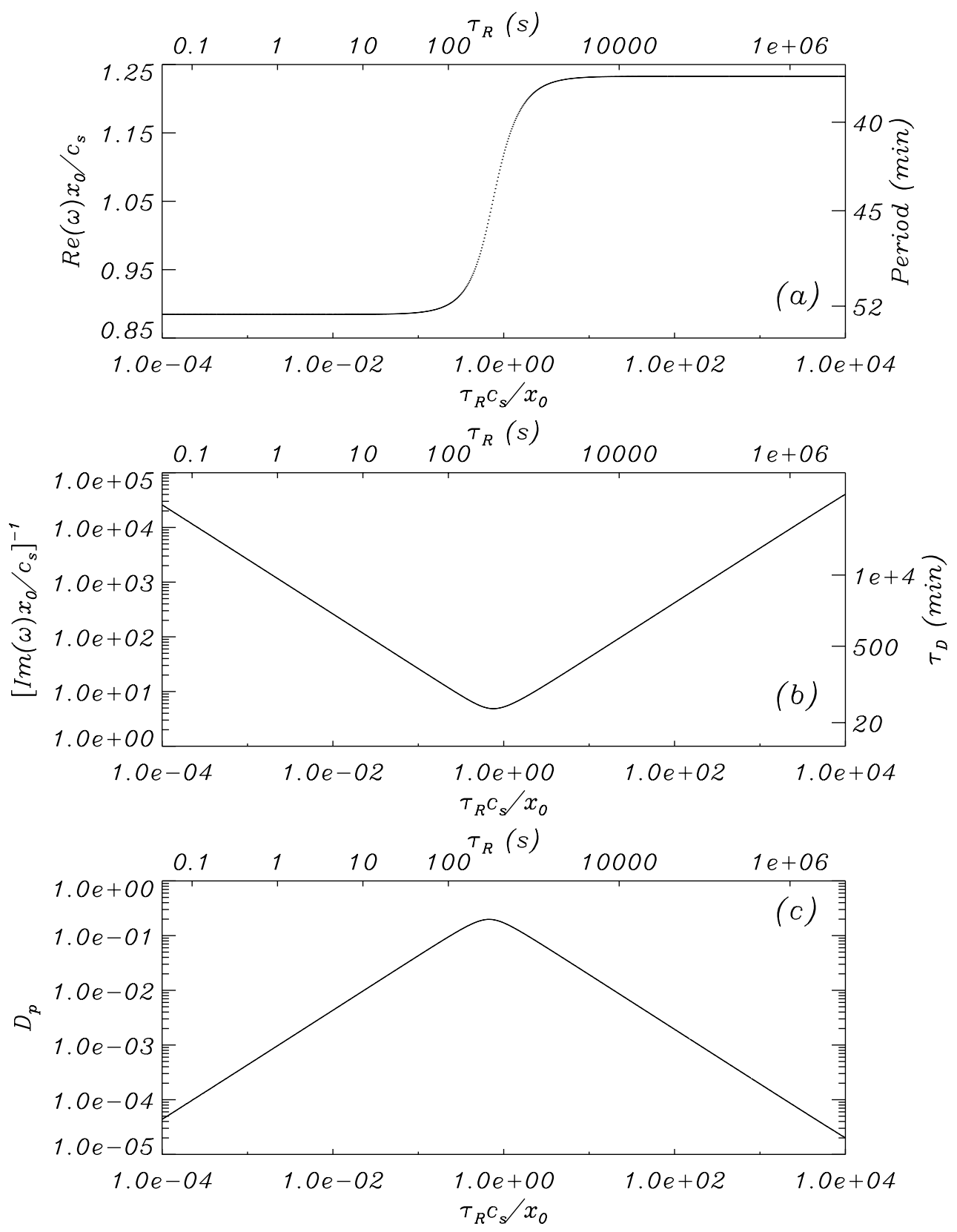

Fig. 5. Plot of some wave parameters versus the radiative relaxation time, $\tau_{\mathrm{R}}$, in Menzel's prominence model: a) real part of the frequency, b) inverse of the imaginary part of the frequency (i.e. damping time) and c) damping per period, $D_{\mathrm{p}}=\operatorname{Im}(\omega) / \operatorname{Re}(\omega)$, for the fundamental slow mode with $k_{z} x_{0}=2$.

increases and tends to infinity as expected. Also, Fig. 5c shows that the maximum of the damping per period occurs for a value of $\tau_{\mathrm{R}}$ close to the minimum of the damping time.

In Fig. 6 we have plotted the same quantities as in Fig. 5 but now for a smaller value of the vertical wavenumber. Now, the transition from isothermal to adiabatic perturbations is slightly different and the period displays a strong jump around $\tau_{\mathrm{R}}=330 \mathrm{~s}$, reaching a maximum value (well above $200 \mathrm{~min}$ ) which is larger than that attained in the isothermal limit. Also, both the damping time and the damping per period show non-symmetric profiles around $\tau_{\mathrm{R}}=330 \mathrm{~s}$. This particular behaviour of the fundamental slow mode at low $k_{z}$ is caused by the destabilising action of gravity, which becomes dominant for low $k_{z}$ and $k_{x}$ (and thus for small $\omega$ ). In fact, this effect is closely related to a situation in which the slow mode can become unstable and which is discussed in Appendix B with the help of a vertically stratified configuration with horizontal magnetic field. An important consequence of the behaviour shown in Figs. $6 \mathrm{a}$ and $6 \mathrm{~b}$ is that certain combinations of low $k_{z}$ and radiative relaxation time $\left(\tau_{\mathrm{R}}\right)$ can give place to slow modes with long periods and short damping times.

In Fig. 7 we have plotted the same parameters as before but for the fundamental fast mode. Now, the 


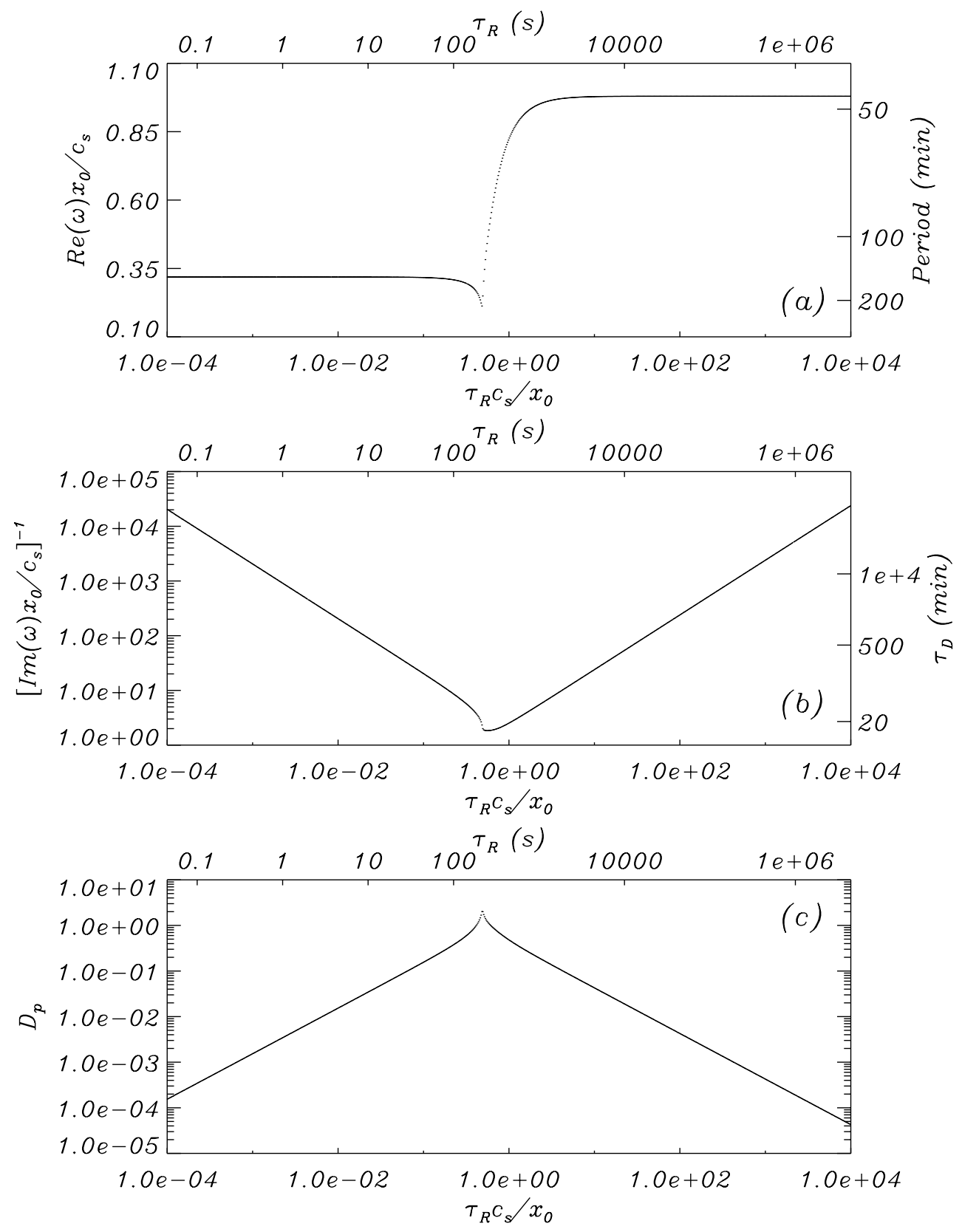

Fig. 6. Plot of some wave parameters versus the radiative relaxation time, $\tau_{\mathrm{R}}$, in Menzel's prominence model: a) real part of the frequency, b) inverse of the imaginary part of the frequency (i.e. damping time) and c) damping per period, $D_{\mathrm{p}}=\operatorname{Im}(\omega) / \operatorname{Re}(\omega)$, for the fundamental slow mode with $k_{z} x_{0}=0.2$.

transition between the isothermal and adiabatic limits occurs at a different value of $\tau_{\mathrm{R}}$ with respect to the slow mode, the change in the real and imaginary parts of the frequency is quite small and the maximum damping time is approximately ten times the period, so the effect of the dissipation mechanism on fast modes is almost negligible.

Now, the computed $D_{\mathrm{p}}$ can be compared with the values obtained by Molowny-Horas et al. (1999), which are in the range $10^{-2}-10^{-1}$. For the fundamental slow mode, Figs. $5 \mathrm{c}$ and $6 \mathrm{c}$ show that the damping per period lies between $10^{-2}$ and $10^{-1}$ for a wide range of values of the radiative relaxation time $\left(10 \mathrm{~s} \leq \tau_{\mathrm{R}} \leq 10^{4} \mathrm{~s}\right)$, for which there is a strong damping of oscillations. On the contrary, the fundamental fast mode is not so strongly damped and $D_{\mathrm{p}}$ is around $10^{-2}$ at most (Fig. $7 \mathrm{c}$ ).

On the other hand, the maximum value of the plasma pressure, density and temperature perturbations has also been plotted against the radiative relaxation time (Figs. 8 and 9$)$. To evaluate these three parameters, $p_{1}(x), \rho_{1}(x)$ and $T_{1}(x)$ have been computed from Eqs. (23)-(25) and the maximum value of their modulus, which gives an idea of the strength of these perturbations, has been kept. From Fig. 8 we notice that, in the adiabatic and isothermal limits, the perturbed pressure modulus is equal to perturbed density plus perturbed temperature moduli, which implies that time variations of the three physical variables 

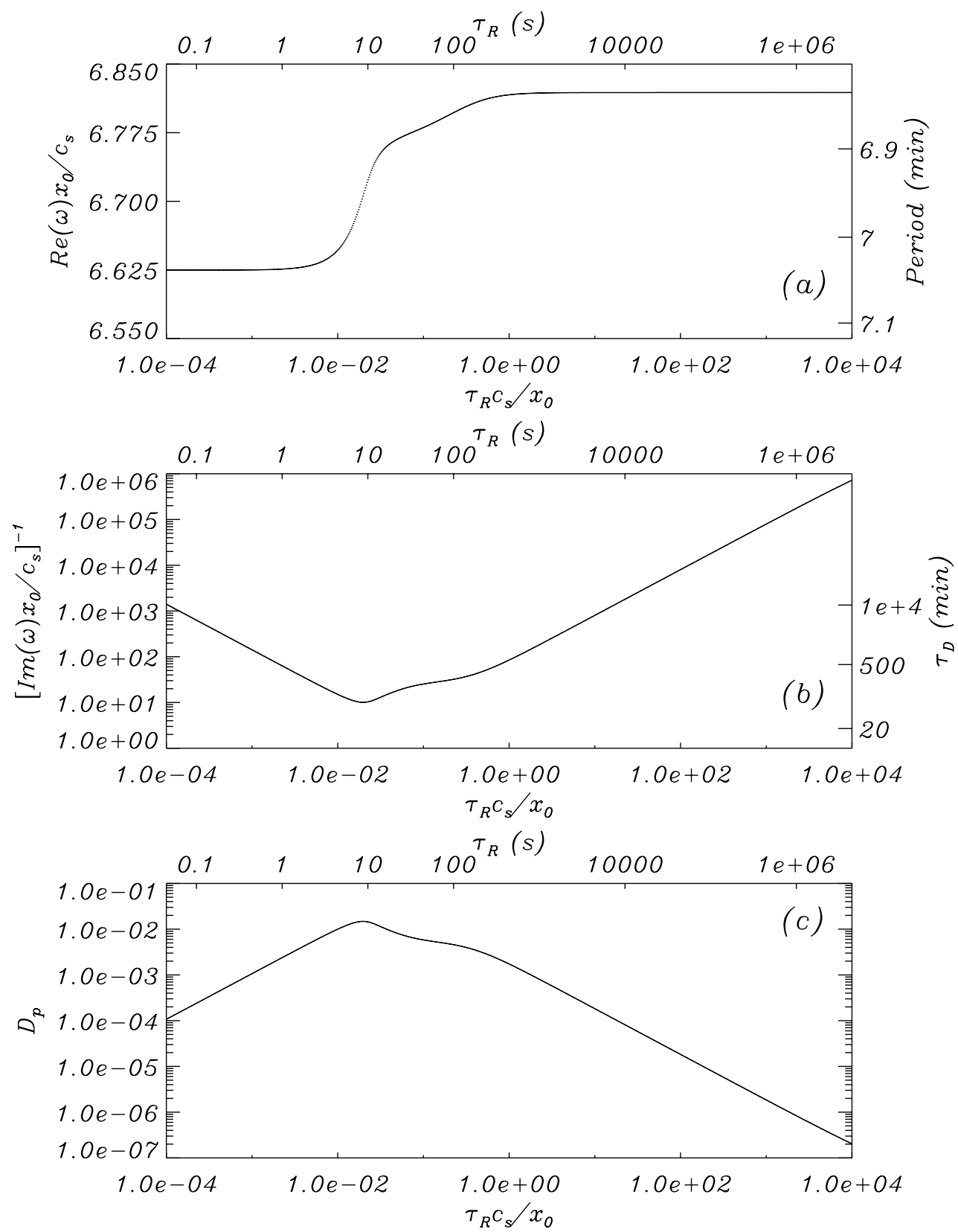

Fig. 7. Plot of some wave parameters versus the radiative relaxation time, $\tau_{\mathrm{R}}$, in Menzel's prominence model: a) real part of the frequency, b) inverse of the imaginary part of the frequency (i.e. damping time) and c) damping per period, $D_{\mathrm{p}}=\operatorname{Im}(\omega) / \operatorname{Re}(\omega)$, for the fundamental fast mode with $k_{z} x_{0}=0.2$.

are in phase. This not the case for intermediate values of $\tau_{\mathrm{R}}$, for which these variables are out of phase. It is also interesting to point out the behaviour of temperature variations versus the radiative relaxation time (see Fig. 8c). Temperature perturbations attain the same amplitude in the range of $\tau_{\mathrm{R}}$ between $\infty$ and $330 \mathrm{~s}$ and, for smaller values of this parameter, their amplitude decreases exponentially with $\tau_{\mathrm{R}}$ such as corresponds to isothermal disturbances. This allows to make a clear distinction between the adiabatic and isothermal regimes. On the other hand, since the results indicate that the oscillatory parameters are greatly influenced by the vertical wavenumber and it is not easy to infer the value of $k_{z}$ from observational data, we have considered a typical prominence height $H=5 \times 10^{4} \mathrm{~km}$ and have taken $\lambda_{z}=2 H$, so that $k_{z}=\pi / H$ or $k_{z} x_{0}=0.33$. Then, for this vertical wavenumber, we can calculate the period and the maximum damping time for the fundamental slow mode, the first slow harmonic and the fundamental fast mode, in both the Kippenhahn-Schlüter and Menzel's equilibria (Table 1). The first conclusion is that, in both configurations, fast modes do not suffer time damping while slow modes are strongly affected by the considered dissipation mechanism. In particular, for a Kippenhahn-Schlüter 

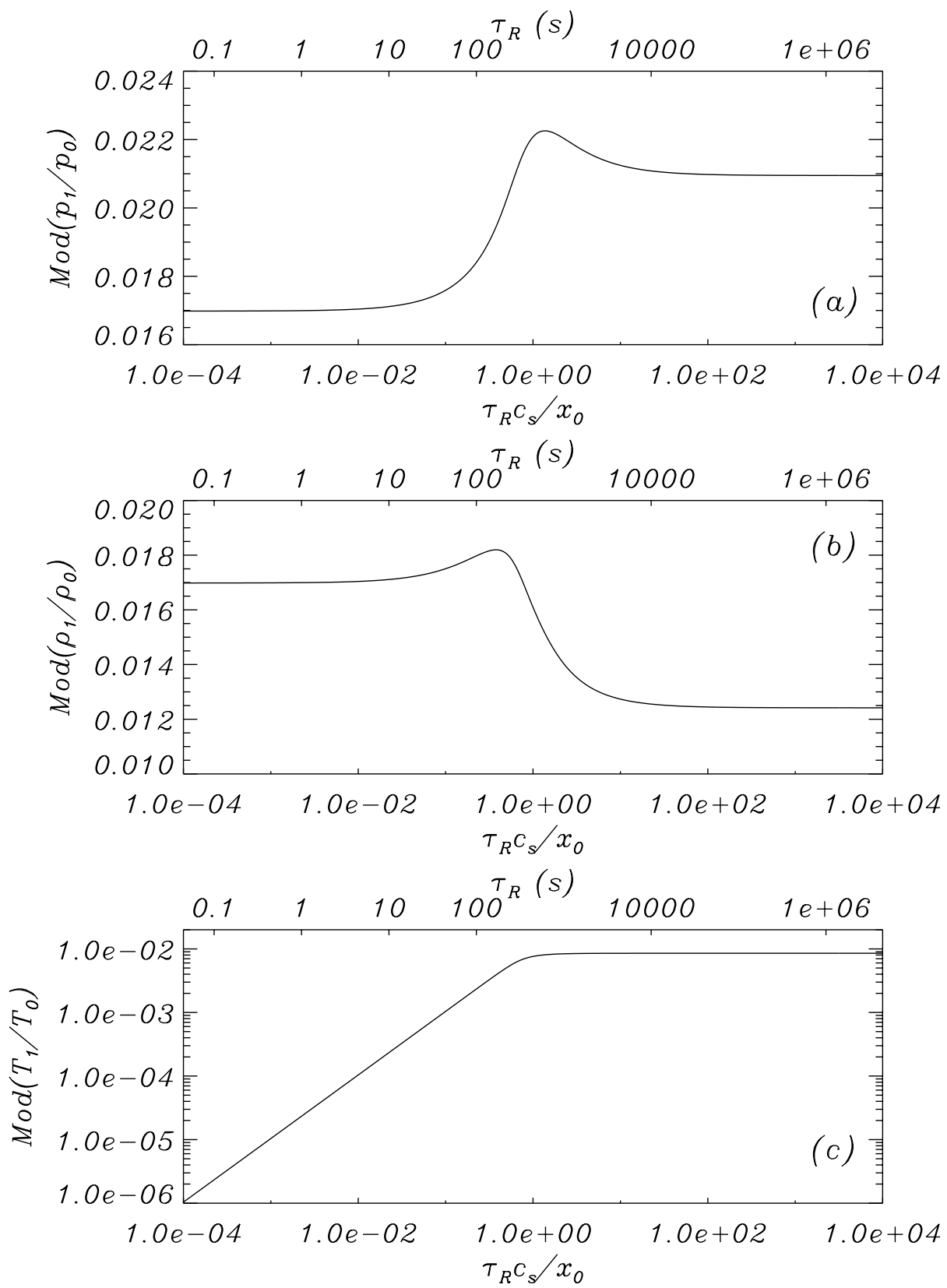

Fig. 8. Menzel's prominence model: Behaviour of the pressure, density and temperature perturbations, normalised to their equilibrium values, versus the radiative relaxation time, $\tau_{\mathrm{R}}$, for the fundamental slow mode. Maximum modulus of a) $p_{1}(x)$, b) $\rho_{1}(x)$ and c) $T_{1}(x)$.

prominence, the fundamental slow mode is practically undamped while the first harmonic is strongly damped. However, in Menzel's prominence model the fundamental mode as well as its harmonics are all strongly damped. The difference in the period and damping time for the fundamental slow mode in Menzel's equilibrium with $q=14$ and $q=18$ is due to their different scale height which makes the effect of gravity less significant for $q=18$. To analyse in more detail the damping times of slow modes in both equilibria, we have plotted the minimum value of the damping time, $\tau_{\text {Dmin }}$, and the corresponding radiative time, $\tau_{\mathrm{Rmin}}$, versus $k_{z}$ (see Figs. 10 and 11). In both models, $\tau_{\text {Dmin }}$ and $\tau_{\text {Rmin }}$ show more dependence on $k_{z}$ for the fundamental slow mode than for its harmonics. In particular, for Menzel's model there is a great difference between the values of damping time for high and low values of the vertical wavenumber, this difference being caused by gravity's effect such as was pointed out before. Also, notice that all the harmonics have similar values for $\tau_{\text {Dmin }}$ and $\tau_{\text {Rmin }}$ in both equilibria.

\subsection{Mode coupling}

Next, we have constructed three-dimensional dispersion diagrams by plotting the real and imaginary part of $\omega$ versus $k_{z}$. In the adiabatic limit (e.g. Paper I and 

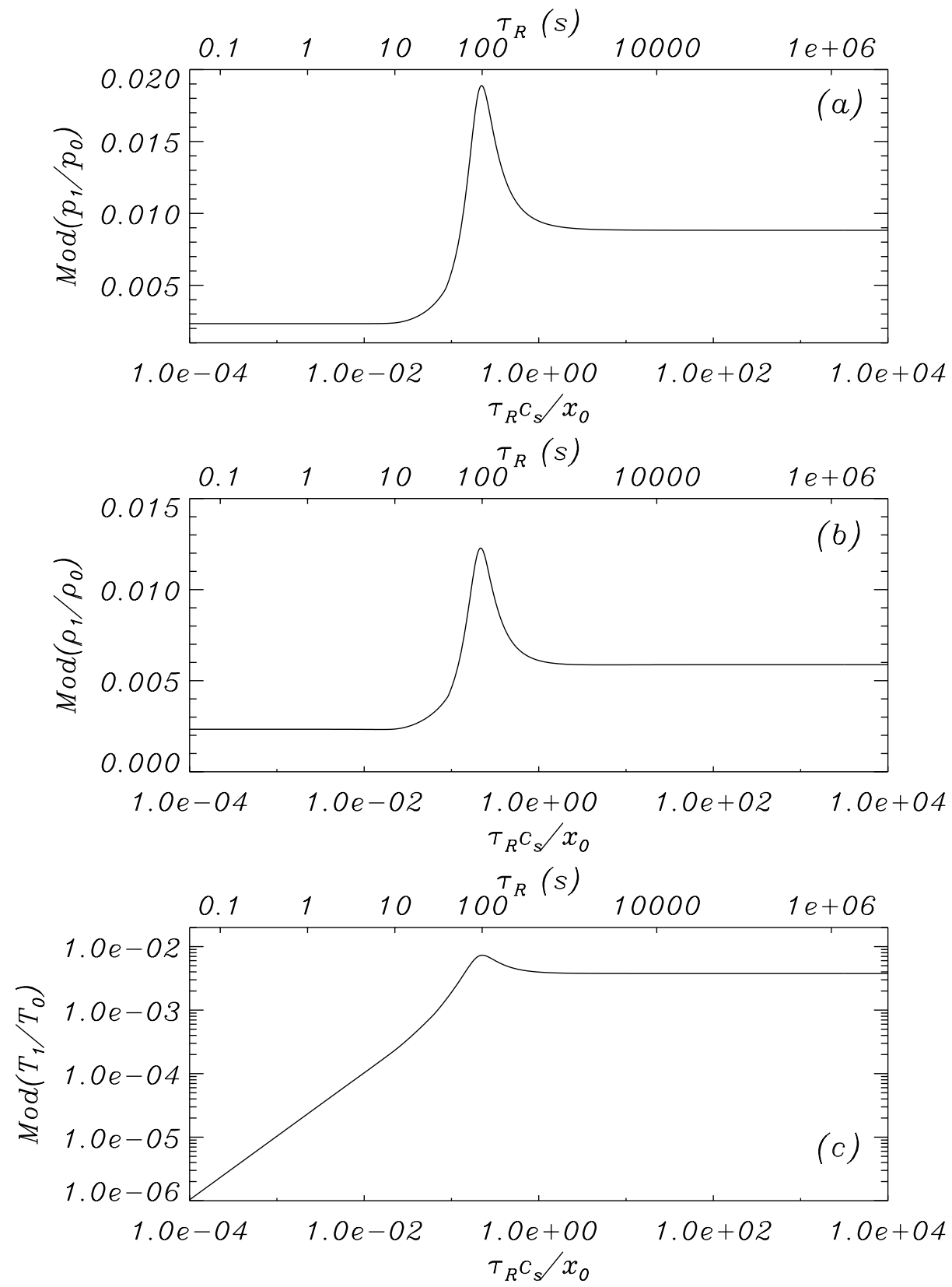

Fig. 9. Menzel's prominence model: Behaviour of the pressure, density and temperature perturbations, normalised to their equilibrium values, versus the radiative relaxation time, $\tau_{\mathrm{R}}$, for the fundamental fast mode. Maximum modulus of a) $p_{1}(x)$, b) $\rho_{1}(x)$ and c) $T_{1}(x)$.

Joarder \& Roberts 1993a), the dispersion diagrams show "avoided crossings" between the two magnetoacoustic modes, i.e. regions in the diagnostic diagram where a strong coupling between the modes takes place and the character of solutions is a mixture of both modes. Now, the effect of Newtonian cooling on these avoided crossings deserves attention. As can be seen in Fig. 12a, the imaginary part of $\omega$ has quite different values for slow and fast modes. Near the avoided crossing the modes interchange their behaviour and slow modes are transformed into fast modes and vice versa. As the value of $\tau_{\mathrm{R}}$ is decreased, solutions are damped in time and so the imaginary part of the frequency increases, but now the two modes no longer exchange their properties as $k_{z}$ is varied in the diagnostic diagram following slow and fast branches (Fig. 12b). In fact, there is only a minor sign of coupling between fast and slow modes around $k_{z} x_{0}=3.6$. Newtonian cooling can even suppress modes coupling for certain values of $\tau_{R}$ (Fig. 12c). In the isothermal limit $\left(\tau_{\mathrm{R}} \ll 1\right)$, the imaginary parts of the frequency cross again (Fig. 12d) in a similar way as in Fig. 12a, until the imaginary part disappears in the purely isothermal case, as expected. Banerjee et al. (1997) found a similar behaviour for the imaginary part of $\omega$ in the case of a bounded vertical magnetic field with 


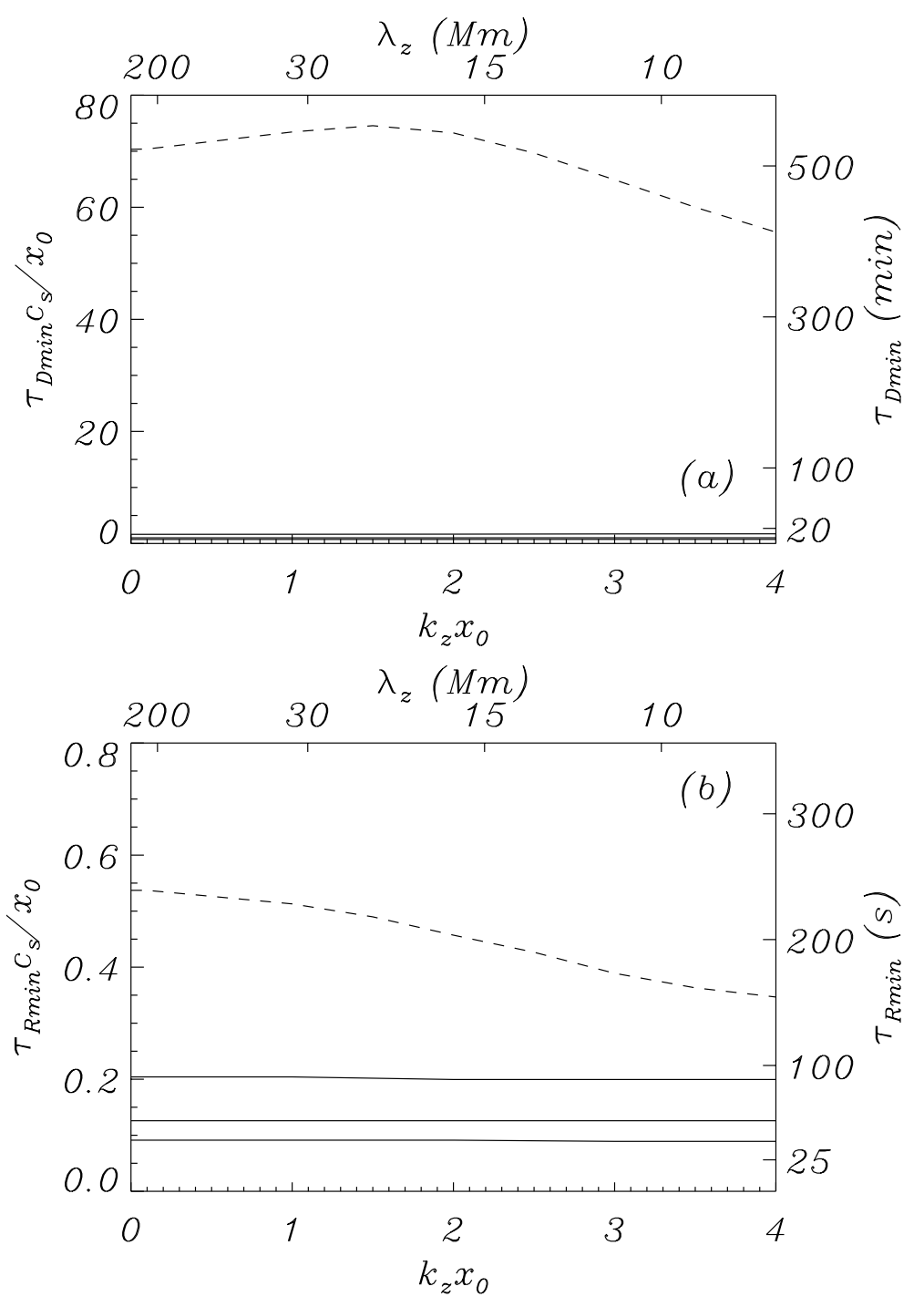

Fig. 10. Kippenhahn-Schlüter model: a) Minimum value of the damping time, $\tau_{\text {Dmin }}$, and $\mathbf{b}$ ) radiative relaxation time for which $\tau_{\text {Dmin }}$ is attained, $\tau_{\text {Rmin }}$, versus the dimensionless vertical wavenumber. Dashed and solid lines correspond to the fundamental slow mode and its first harmonics, respectively.

gravity and Christensen-Dalsgaard (1981) obtained a similar result studying non radial oscillations in the case of non adiabatic waves.

\section{Conclusions}

As a first approach to explain the time damping detected in observations of quiescent prominence oscillations, we have considered a radiative loss term, represented by Newton's law of cooling, in the system of linearised equations describing the oscillations of two different quiescent prominence models. The main conclusions we have extracted from this study are:

1. In the Kippenhahn-Schlüter model, the fundamental slow mode is almost undamped while its harmonics are strongly damped. On the opposite, when a Menzel's prominence model is considered, all slow modes display strong time damping.
2. Two values of the the parameter $q$ in Menzel's model, namely $q=14$ and $q=18$, have been considered. It has been found that when $q$ is increased (that is, when the curvature of magnetic field lines is increased) the oscillatory period of the fundamental slow mode for intermediate values of $\tau_{\mathrm{R}}$ decreases, while the damping time increases.

3. In both equilibrium models, fast modes are practically undamped and their frequency is not seriously modified by the damping mechanism.

4. When Menzel's model is considered, high values for the oscillation periods are obtained in the regime of small vertical wavenumbers. Then, some combinations of low vertical wavenumbers and $\tau_{\mathrm{R}}$ give place to slow modes having long periods and short damping times.

5. Since velocity perturbations corresponding to slow modes are polarised in the horizontal direction perpendicular to the prominence sheet (in equilibrium configurations with transverse magnetic field such as 


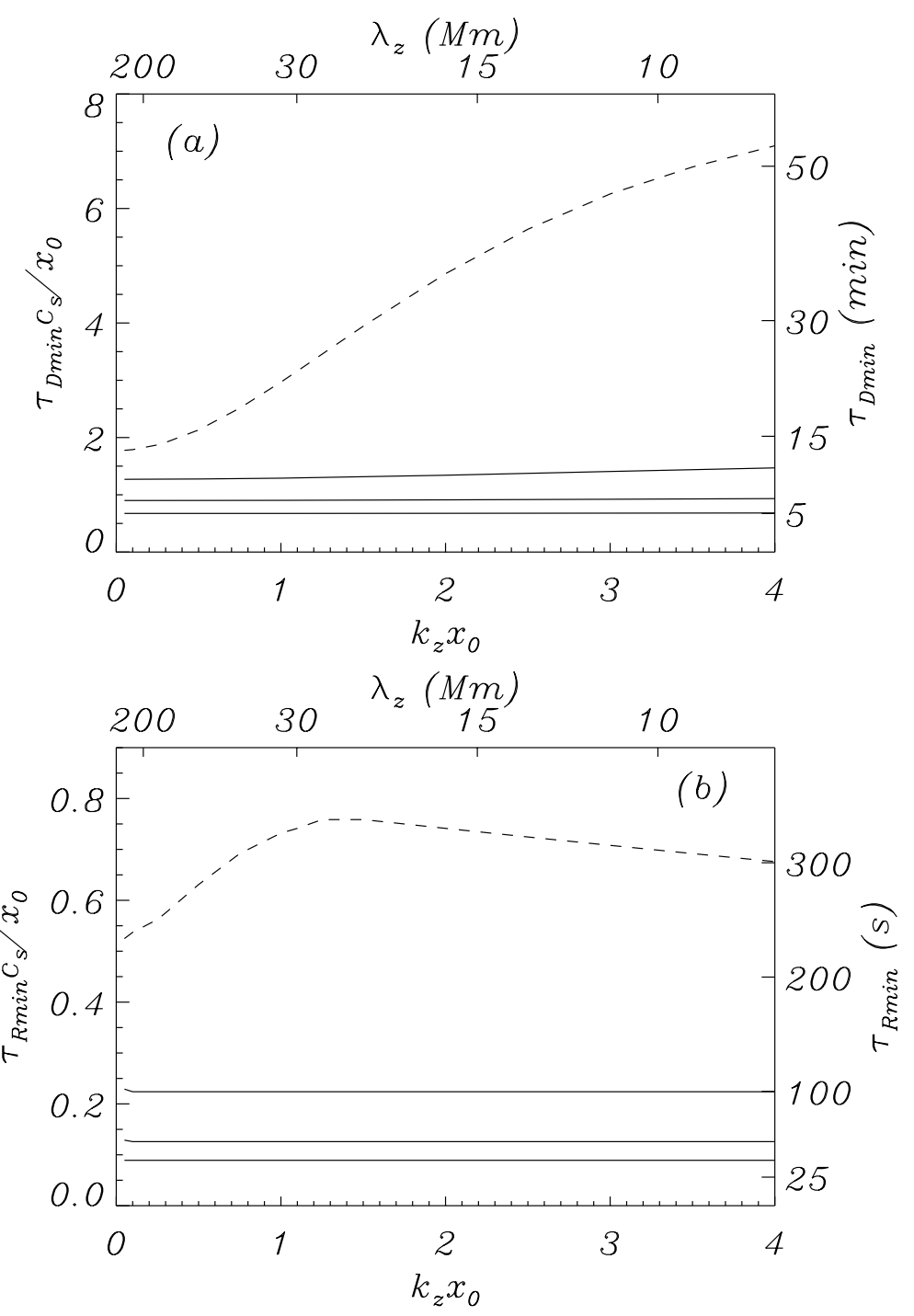

Fig. 11. Menzel's prominence model: a) Minimum value of the damping time, $\tau_{\text {Dmin }}$, and $\mathbf{b}$ ) radiative relaxation time for which $\tau_{\text {Dmin }}$ is attained, $\tau_{\text {Rmin }}$, versus the dimensionless vertical wavenumber. Dashed and solid lines correspond to the fundamental slow mode and its first harmonics, respectively.

the ones considered here), the damping of oscillations should be more easily observed in prominences at the limb, such as in Molowny-Horas et al. (1999), and not in filaments observed on the disk.

6. The behaviour of the three-dimensional dispersion diagrams has been studied and the peculiar effect of the radiative losses on the avoided crossings of the magnetoacoustic modes has been pointed out.

7. Such as was pointed out in the Introduction, another mode is present in both equilibrium configurations, the thermal mode. The frequency of this mode is purely imaginary, i.e. it is a purely time damped mode (see Webb \& Roberts 1980). However, we are not interested in this mode because it does not represent propagating waves.

The considered damping mechanism only allows to explain the time damping of slow modes, and it would be necessary to resort to a different mechanism to explain the damping of fast modes. A possibility is to consider the fibril structure of prominences, with fibrils modeled as thin flux tubes, and to study the leakage of energy of fast modes confined within these fibrils (see Joarder et al. 1997; Díaz et al. 2001). Then, different damping mechanisms could be working at the same time, which would allow to explain the damping of the different magnetoacoustic modes. On the other hand, it is difficult to clearly identify the different modes of oscillation from observations of prominence oscillations. For instance, in the case of a sheared prominence, the coupling of slow, fast and Alfvén modes is expected to produce important modifications in the velocity perturbations characterising each of those modes (Joarder \& Roberts 1993b).

Finally, the damping of perturbations is probably a common feature of prominence oscillations, so this theoretical line should be developed further by exploring more 

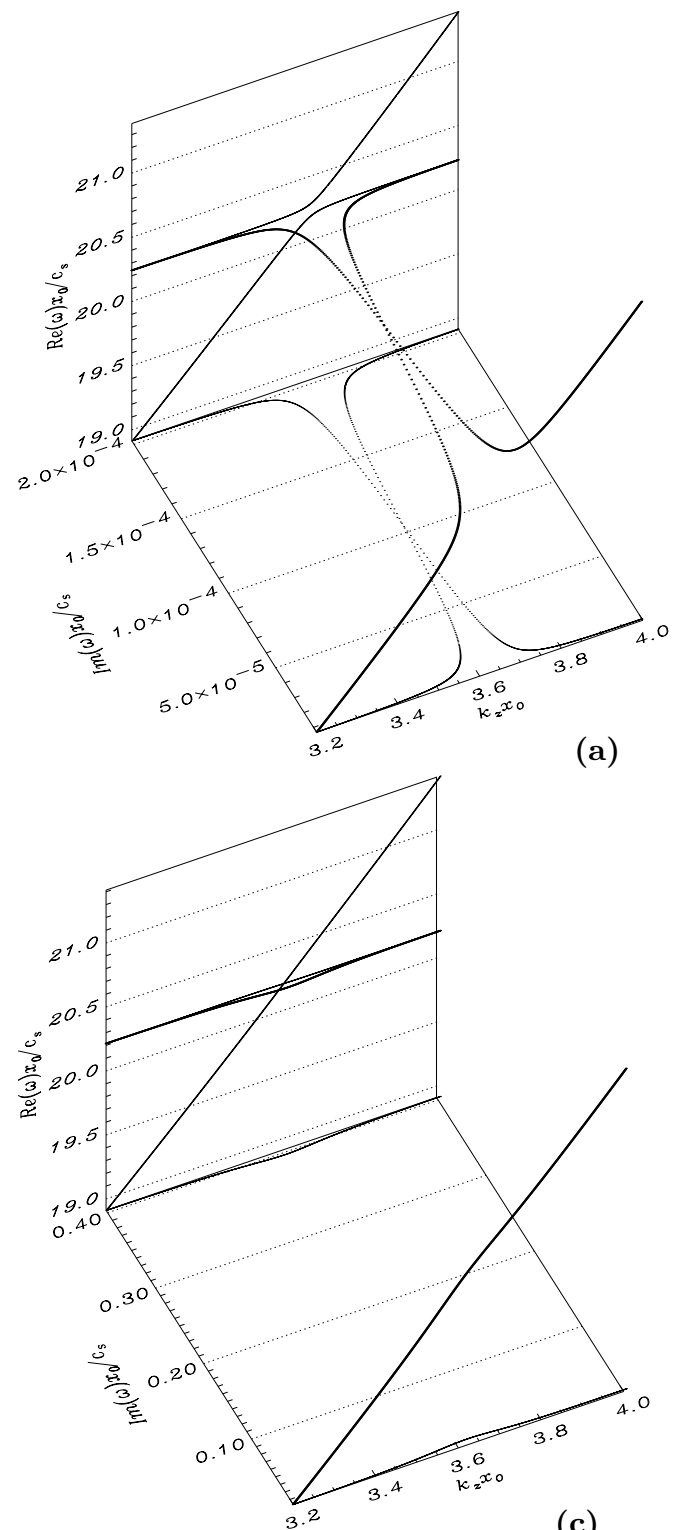

(c)
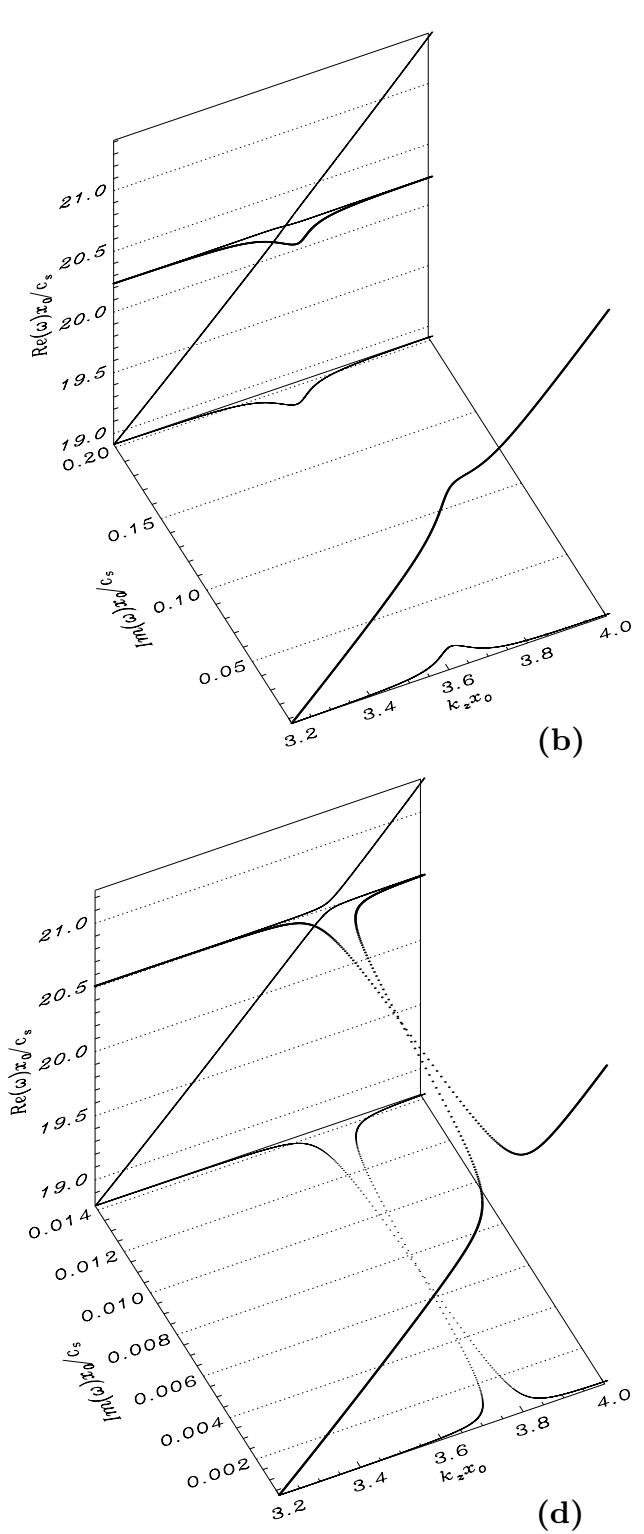

(d)

Fig. 12. Three-dimensional dispersion diagrams showing the effect of Newton's law of cooling at the "avoided crossings" between a slow and a fast mode for a) $\left.\left.\left.\tau_{\mathrm{R}}=4.5 \times 10^{6} \mathrm{~s}, \mathbf{b}\right) \tau_{\mathrm{R}}=445 \mathrm{~s}, \mathbf{c}\right) \tau_{\mathrm{R}}=222 \mathrm{~s}, \mathbf{d}\right) \tau_{\mathrm{R}}=0.04 \mathrm{~s}$.

sophisticated energy equations allowing to study the effect of radiation, conduction and heating on prominence oscillations, the effect of wave leakage, resonant absorption, etc.

Acknowledgements. The authors want to acknowledge the financial support received from MCyT under grant BFM20001329.

\section{Appendix A: Coefficients of the governing differential equations}

The coefficients of the two second order differential equations (Eqs. (28) and (29)) are the following,

$q_{1}=-\left[\frac{1}{\rho_{0}}\left(\frac{\partial \rho_{0}}{\partial x}+\frac{B_{0 z}}{B_{0 x}} \frac{\partial \rho}{\partial z}\right)+\frac{B_{0 z}}{B_{0 x}} \frac{g}{\hat{c}_{\mathrm{s}}^{2}}-i k_{z} \frac{B_{0 z}}{B_{0 x}}\right]$

$$
q_{2}=\frac{1}{\hat{c}_{\mathrm{s}}^{2}}\left(i k_{z} \hat{c}_{\mathrm{s}}^{2}+g\right)
$$

$$
\begin{aligned}
& q_{3}=-\frac{\omega^{2}}{\hat{c}_{\mathrm{s}}^{2}} \\
& q_{4}=-\frac{\omega^{2}}{\hat{c}_{\mathrm{s}}^{2}} \frac{B_{0 z}}{B_{0 x}}+k_{z}^{2} \frac{B_{0 z}}{B_{0 x}}+i k_{z} \frac{1}{\rho_{0}}\left(\frac{\partial \rho_{0}}{\partial x}+\frac{B_{0 z}}{B_{0 x}} \frac{\partial \rho_{0}}{\partial z}\right)
\end{aligned}
$$

$$
\begin{aligned}
q_{5}= & -\frac{\mu \rho_{0}}{B_{0 x}^{2}}\left\{\hat{c}_{\mathrm{s}}^{2} \frac{1}{\rho_{0}} \frac{\partial \rho_{0}}{\partial z}-2 \frac{B_{0 x}}{\mu \rho_{0}} \frac{\partial B_{0 z}}{\partial x}+g\right. \\
& \left.+\frac{B_{0 x} B_{0 z}}{\mu \rho_{0}}\left[\frac{1}{\rho_{0}}\left(\frac{\partial \rho_{0}}{\partial x}+\frac{B_{0 z}}{B_{0 x}} \frac{\partial \rho_{0}}{\partial z}\right)+\frac{B_{0 z}}{B_{0 x}} \frac{g}{\hat{c}_{\mathrm{s}}^{2}}\right]\right\} \\
& +i k_{z} \frac{\mu \rho_{0}}{B_{0 x}^{2}}\left(\hat{c}_{\mathrm{s}}^{2}+\frac{B_{0 z}^{2}}{\mu \rho_{0}}\right),
\end{aligned}
$$


Table 1. Oscillatory periods, maximum damping times and corresponding radiative relaxation times for the KippenhahnSchlüter and Menzel's equilibrium models. All the solutions have been computed with $k_{z} x_{0}=0.33$.

\begin{tabular}{cccccccccc}
\hline \hline Equilibrium & \multicolumn{3}{c}{ Fundamental slow } & \multicolumn{3}{c}{ First harmonic slow } & \multicolumn{3}{c}{ Fundamental fast } \\
& $T(\min )$ & $\tau_{\mathrm{D}}(\mathrm{min})$ & $\tau_{\mathrm{R}}(\mathrm{s})$ & $T(\min )$ & $\tau_{\mathrm{D}}(\mathrm{min})$ & $\tau_{\mathrm{R}}(\mathrm{s})$ & $T(\min )$ & $\tau_{\mathrm{D}}(\min )$ & $\tau_{\mathrm{R}}(\mathrm{s})$ \\
\hline \hline \multirow{3}{*}{$K-S$} & 24.9 & $\infty$ & $\infty$ & 9.8 & $\infty$ & $\infty$ & 6.1 & $\infty$ & $\infty$ \\
& 25.2 & 525 & 223 & 11.1 & 12 & 89 & 6.1 & 133 & 58 \\
& 25.3 & $\infty$ & 0 & 12.6 & $\infty$ & 0 & 6.1 & $\infty$ & 0 \\
\hline \multirow{3}{*}{ Menzel $(q=14)$} & 47.2 & $\infty$ & $\infty$ & 11.4 & $\infty$ & $\infty$ & 6.8 & $\infty$ & $\infty$ \\
& 88.9 & 14 & 267 & 13.7 & 9.4 & 97 & 6.8 & 52 & 9 \\
& 134.9 & $\infty$ & 0 & 16.5 & $\infty$ & 0 & 6.9 & $\infty$ & 0 \\
\hline \multirow{3}{*}{ Menzel $(q=18)$} & 42 & $\infty$ & $\infty$ & 11.1 & $\infty$ & $\infty$ & 6.6 & $\infty$ & $\infty$ \\
& 51.1 & 40 & 312 & 13.2 & 10 & 96 & 6.6 & 68 & 8 \\
& 59.1 & $\infty$ & 0 & 15.7 & $\infty$ & 0 & 6.8 & $\infty$ & 0 \\
\hline
\end{tabular}

$$
\begin{aligned}
q_{6}= & \frac{1}{\hat{c}_{\mathrm{s}}^{2}} \frac{B_{0 z}}{B_{0 x}}\left(i k_{z} \hat{c}_{\mathrm{s}}^{2}+g-\hat{c}_{\mathrm{s}}^{2} \frac{2}{B_{0 z}} \frac{\partial B_{0 x}}{\partial x}\right), \\
q_{7}= & -\frac{\omega^{2}}{\hat{c}_{\mathrm{s}}^{2}} \frac{B_{0 z}}{B_{0 x}}-\frac{B_{0 z}}{B_{0 x}} k_{z}^{2}-i k_{z} \frac{2}{B_{0 x}} \frac{\partial B_{0 z}}{\partial z}, \\
q_{8}= & k_{z}^{2} \frac{\mu \rho_{0}}{B_{0 x}^{2}}\left(\hat{c}_{\mathrm{s}}^{2}+\frac{B_{0 x}^{2}}{\mu \rho_{0}}+\frac{B_{0 z}^{2}}{\mu \rho_{0}}\right) \\
& +i k_{z} \frac{\mu \rho_{0}}{B_{0 x}^{2}}\left\{\hat{c}_{\mathrm{s}}^{2} \frac{1}{\rho_{0}} \frac{\partial \rho_{0}}{\partial z}+2 \frac{B_{0 x}}{\mu \rho_{0}} \frac{\partial B_{0 z}}{\partial x}\right. \\
& \left.+\frac{B_{0 x} B_{0 z}}{\mu \rho_{0}}\left[\frac{1}{\rho_{0}}\left(\frac{\partial \rho_{0}}{\partial x}+\frac{B_{0 z}}{B_{0 x}} \frac{\partial \rho_{0}}{\partial z}\right)\right]\right\} \\
& -\frac{\omega^{2}}{\hat{c}_{\mathrm{s}}^{2}} \frac{\mu \rho_{0}}{B_{0 x}^{2}}\left(\hat{c}_{\mathrm{s}}^{2}+\frac{B_{0 z}^{2}}{\mu \rho_{0}}\right) \cdot
\end{aligned}
$$

\section{Appendix B: Instability conditions}

In the case of Menzel's prominence configuration, a better understanding of some properties of slow modes with small $k_{z}$ (in particular, the variation of $\omega$ with $\tau_{\mathrm{R}}$ in Fig. 6) can be obtained by considering a simple structure for which the wave equations can be treated analytically. We consider a horizontal magnetic field in an isothermal, stratified medium (that is, curvature effects are removed) and we assume that the system is unbounded in the $x$ - and $z$-directions. In this case Eqs. (28)-(29) result in the well known quadratic dispersion relation in $\omega^{2}$,

$$
\begin{aligned}
& \omega^{4}-\left(c_{\mathrm{s}}^{2}+v_{\mathrm{A}}^{2}\right)\left(k^{2}+\frac{1}{4 H_{\mathrm{B}}^{2}}\right) \omega^{2} \\
& +v_{\mathrm{A}}^{2} c_{\mathrm{s}}^{2} k_{x}^{2}\left(k^{2}+\frac{1}{4 H_{\mathrm{B}}^{2}}\right)+g\left(\frac{c_{\mathrm{s}}^{2}}{H_{\mathrm{B}}}-g\right) k_{x}^{2}=0
\end{aligned}
$$

where $k^{2}=k_{x}^{2}+k_{z}^{2}$.

Equation (B.1) indicates that modes become unstable, i.e. $\omega^{2}$ becomes negative, when the independent term is negative. This is the condition for magnetic buoyancy instability, which is satisfied when the horizontal wavenumber, $k_{x}$, is smaller than a critical value, $k_{x \max }$,

$k_{x}^{2}<k_{x \max }^{2}=-\left(k_{z}^{2}+\frac{1}{4 H_{\mathrm{B}}^{2}}\right)+\frac{1}{v_{\mathrm{A}}^{2} c_{\mathrm{s}}^{2}} g\left(g-\frac{c_{\mathrm{s}}^{2}}{H_{\mathrm{B}}}\right)$.
To have instability, the second term on the right-hand side of this expression must necessarily be positive. Using Eq. (13) for the magnetically modified scale height, a necessary condition to be satisfied is

$\frac{v_{\mathrm{A}}^{2}}{c_{\mathrm{s}}^{2}}>2\left(\frac{\gamma-1}{\gamma}\right)=0.8$

or in terms of the plasma beta

$\beta<\frac{1}{\gamma-1}=1.5$

which is the condition found by Pintér et al. (2000), who analysed a similar equilibrium configuration in which the Alfvén mode is present and $k_{y} \neq 0$. This result implies that only strong magnetic fields can be unstable. Equation (B.3) is a necessary but not sufficient condition to have instability because the first term on the righthand side of Eq. (B.2) shows a dependence on the vertical wavenumber and thus only vertical wavenumbers smaller than a critical value give rise to instability. Then, to have instability,

$k_{z}^{2}<k_{z \max }^{2}=-\frac{1}{4 H_{\mathrm{B}}^{2}}+\frac{1}{v_{\mathrm{A}}^{2} c_{\mathrm{s}}^{2}} g\left(g-\frac{c_{\mathrm{s}}^{2}}{H_{\mathrm{B}}}\right)$.

The same arguments can be applied for purely isothermal disturbances. In this case, the adiabatic sound speed $\left(c_{\mathrm{s}}\right)$ must be replaced by the isothermal sound speed $\left(c_{\mathrm{s}} / \sqrt{\gamma}\right)$ in Eqs. (B.2) and (B.5). Now, the critical wave numbers are given by

$k_{x \max }^{2}=-\left(k_{z}^{2}+\frac{1}{4 H_{\mathrm{B}}^{2}}\right)+\frac{1}{v_{\mathrm{A}}^{2} c_{\mathrm{s}}^{2}} g\left(\gamma g-\frac{c_{\mathrm{s}}^{2}}{H_{\mathrm{B}}}\right)$

and

$k_{z \max }^{2}=-\frac{1}{4 H_{\mathrm{B}}^{2}}+\frac{1}{v_{\mathrm{A}}^{2} c_{\mathrm{s}}^{2}} g\left(\gamma g-\frac{c_{\mathrm{s}}^{2}}{H_{\mathrm{B}}}\right)$.

Note that both the critical horizontal and vertical wavenumbers possess larger values than in the adiabatic limit. The isothermal case gives a wider region of instability in the dispersion diagram and condition (B.3) now reduces to

$\frac{v_{\mathrm{A}}^{2}}{c_{\mathrm{S}}^{2}}>2\left(\frac{\gamma-1}{\gamma^{2}}\right)=0.48$. 

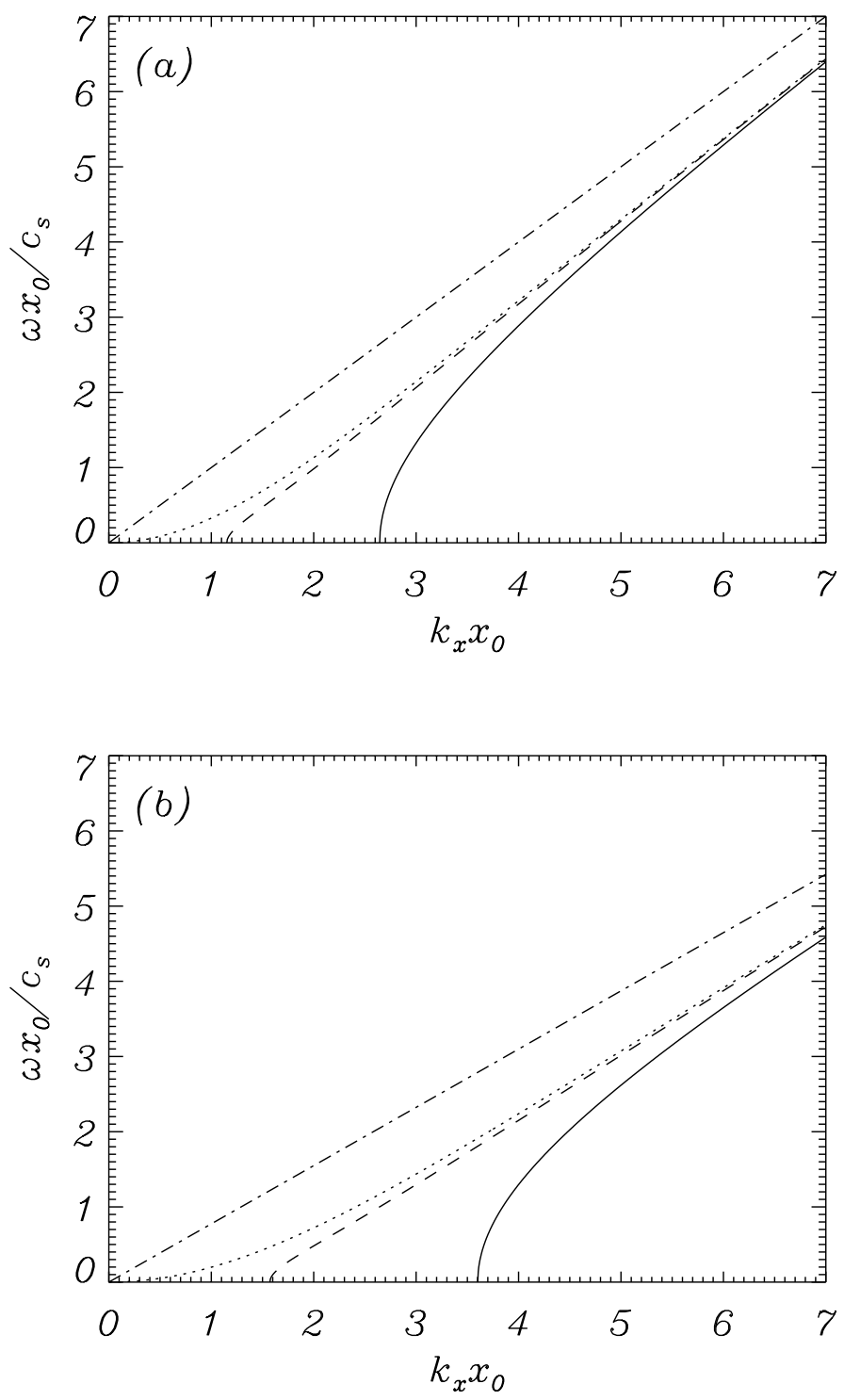

Fig. B.1. Dispersion diagram for the slow mode in an unbounded, vertically stratified atmosphere with horizontal magnetic field. a) Adiabatic perturbations and b) isothermal perturbations. Solid, dashed and dotted lines correspond to $k_{z}=0,0.9 k_{z \max }$ and $k_{z \max }$, see Eqs. (B.5) and (B.7). Dashed-dotted lines correspond to the slow mode in a non stratified equilibrium $\left(H_{\mathrm{B}} \rightarrow \infty\right)$.

Therefore, we conclude that isothermal perturbations are more unstable than adiabatic ones, and magnetic fields that are stable in the adiabatic case can become unstable in the isothermal limit.

Next, dispersion diagrams of the slow mode have been obtained by solving Eq. (B.1) for adiabatic and isothermal perturbations (see Fig. B.1). In these plots, values of the vertical wavenumber between 0 , which results in the maximum value of $k_{x}$ for which there is instability, and $k_{z \max }$ have been used. According to Eqs. (B.2) and (B.6), the instability domain for each $k_{z} \leq k_{z \max }$ consists of the region to the left of each curve in Figs. B.1a and b. It is interesting to note that for high values of $k_{x}$ the frequency tends to that of a slow mode in a uniform medium.

In the present unbounded medium all values of $k_{x}$ are admissible and those satisfying $k_{x} \leq k_{x \max }$, with $k_{z} \leq k_{z \max }$, correspond to unstable disturbances. However, in bounded systems like those considered in this paper, the wavenumber is quantised in such a way that different harmonics in the dispersion diagram (such as in Fig. 3) essentially select the appropriate $k_{x}$ so as to satisfy the boundary conditions at $x= \pm x_{0}$. As the order of the harmonic is increased, the effective horizontal wavenumber also increases. Then, taking Fig. B.1 as a guide, the fundamental slow mode in Menzel's configuration must be closer to the instability domain than any of its harmonics and the tendency towards instability must be accentuated for small $k_{z}$. This is the reason for the large values of the period found in Fig. 3.

When temperature disturbances of this unbounded configuration are governed by Newton's law of cooling, the sound speed must be substituted by that given by 


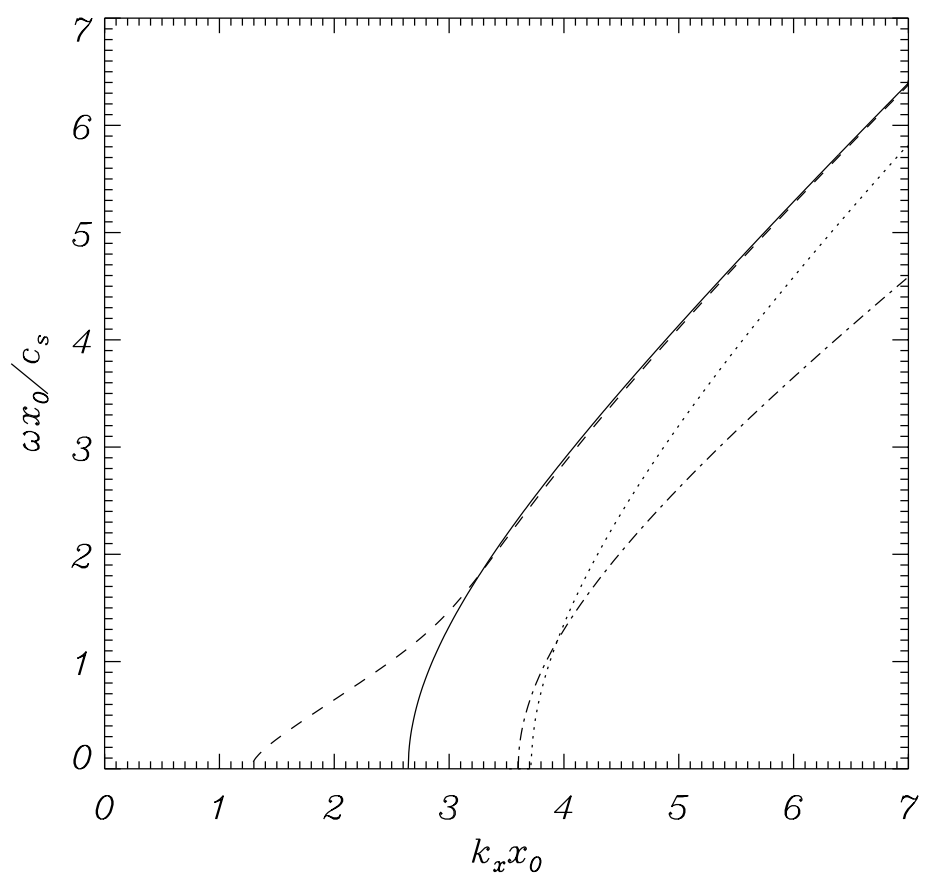

Fig. B.2. Slow mode dispersion diagram in an unbounded, vertically stratified atmosphere with horizontal magnetic field. The frequency has been plotted against $k_{x}$ for the most destabilising vertical wavenumber $\left(k_{z}=0\right)$ and different values of the radiative relaxation time: $\tau_{\mathrm{R}} c_{\mathrm{s}} / x_{0} \rightarrow \infty$ (solid line), $\tau_{\mathrm{R}} c_{\mathrm{s}} / x_{0}=1.2$ (dashed line), $\tau_{\mathrm{R}} c_{\mathrm{s}} / x_{0}=0.2$ (dotted line) and $\tau_{\mathrm{R}} c_{\mathrm{s}} / x_{0}=0$ (dashed-dotted line).

Eqs. (21) and (22) and the dispersion relation becomes

$$
\begin{aligned}
& \omega^{5}-\frac{i}{\tau_{\mathrm{R}}} \omega^{4}-\left(c_{\mathrm{s}}^{2}+v_{\mathrm{A}}^{2}\right)\left(k^{2}+\frac{1}{4 H_{\mathrm{B}}^{2}}\right) \omega^{3} \\
& +\frac{i}{\tau_{\mathrm{R}}}\left(\frac{c_{\mathrm{s}}^{2}}{\gamma}+v_{\mathrm{A}}^{2}\right)\left(k^{2}+\frac{1}{4 H_{\mathrm{B}}^{2}}\right) \omega^{2} \\
& +\left[v_{\mathrm{A}}^{2} c_{\mathrm{s}}^{2} k_{x}^{2}\left(k^{2}+\frac{1}{4 H_{\mathrm{B}}^{2}}\right)+g\left(\frac{c_{\mathrm{s}}^{2}}{H_{\mathrm{B}}}-g\right) k_{x}^{2}\right] \omega \\
& -\frac{i}{\tau_{\mathrm{R}}}\left[v_{\mathrm{A}}^{2} \frac{c_{\mathrm{s}}^{2}}{\gamma} k_{x}^{2}\left(k^{2}+\frac{1}{4 H_{\mathrm{B}}^{2}}\right)+g\left(\frac{c_{\mathrm{s}}^{2}}{\gamma H_{\mathrm{B}}}-g\right) k_{x}^{2}\right]=0 .
\end{aligned}
$$

Now it is possible to study the influence of Newton's law of cooling on the stability properties of the slow mode in the unbounded medium. After selecting the most unstable vertical wavenumber $\left(k_{z}=0\right)$ the slow mode frequency has been computed from Eq. (B.9) for several values of $\tau_{R}$. Figure B.2 reveals that, as one moves from the adiabatic to the isothermal limit, the stability curve does not show a gradual change between the corresponding curves, and so the slow mode does not become progressively more unstable as $\tau_{\mathrm{R}}$ decreases. Instead, the mode first becomes more stable $\left(\tau_{\mathrm{R}} c_{\mathrm{S}} / x_{0}=1.2\right)$ and then, for smaller values of the radiative relaxation time $\left(\tau_{\mathrm{R}} c_{\mathrm{S}} / x_{0}=0.2\right)$, becomes more unstable than in the isothermal limit. Therefore, it is possible to select a horizontal wavenumber, e.g. $k_{x} x_{0} \simeq 3.7$ in Fig. B.2, for which the slow mode is stable in the adiabatic and isothermal limits but is unstable for intermediate values of $\tau_{\mathrm{R}}$. Turning back to radiatively damped MHD perturbations of Menzel's configuration, the above behaviour is basically what we see in Fig. 6a: starting from the adiabatic limit $\left(\tau_{\mathrm{R}} \rightarrow \infty\right)$ the real part of the frequency decreases towards its value in the isothermal regime $\left(\tau_{R}=0\right)$, but for a small range of $\tau_{R}$ the combined effect of gravity and Newton's law of cooling tend to destabilise this slow mode and $\omega$ displays a sharp minimum.

As a final remark, Fig. B.1 clearly shows that gravitational stratification is responsible for the destabilisation of the slow mode and that for $H_{\mathrm{B}} \rightarrow \infty$ (such as in the Kippenhahn-Schlüter model, in which gravity is present but does not give rise to stratification) this mode is always stable. This is in good agreement with Galindo-Trejo \& Schindler (1984), who showed that the KippenhahnSchlüter configuration is stable, and with Galindo-Trejo (1987), who showed that for a certain range of parameters Menzel's equilibrium solution is unstable.

\section{References}

Banerjee, D., Hasan, S. S., \& Christensen-Dalsgaard, J. 1997, Solar Phys., 172, 52

Bünte, M., \& Bogdan, T. J. 1994, A\&A, 283, 642

Christensen-Dalsgaard, J. 1981, A\&A, 283, 642

Díaz, A. J., Oliver, R., \& Ballester, J. L. 2001, A\&A, submitted Galindo-Trejo, J., \& Schindler, K. 1984, ApJ, 277, 422

Galindo-Trejo, J. 1987, Solar Phys., 108, 265

Hood, A. W. 1992, Plasma Phys. Control. Fusion, 34, 4, 411

Joarder, P. S., \& Roberts, B. 1992, A\&A, 261, 625

Joarder, P. S., \& Roberts, B. 1993a, A\&A, 273, 642

Joarder, P. S., \& Roberts, B. 1993b, A\&A, 277, 225 
Joarder, P. S., Nakariakov, V. M., \& Roberts, B. 1997, Solar Phys., 173, 81

Mihalas, D., \& Mihalas, B. W. 1984, in Foundations of Radiation Hydrodynamics (Oxford University Press, New York)

Molowny-Horas, R., Oliver, R., Ballester, J. L., \& Baudin, F. 1997, Solar Phys., 172, 181

Molowny-Horas, R., Wiehr, E., Balthasar, H., Oliver, R., \& Ballester, J. L. 1999, in JOSO Annual Report '98, ed. A. Antalová, H. Balthasar, \& A. Kučera, Astronomical Institute Tatranská Lomnica, 126

Oliver, R., Ballester, J. L., Hood, A. W., \& Priest, E. R. 1992, ApJ, 400, 369, Paper I

Oliver, R., Ballester, J. L., Hood, A. W., \& Priest, E. R. 1993, ApJ, 409, 809

Oliver, R., \& Ballester, J. L. 1995, ApJ, 448, 444

Oliver, R., \& Ballester, J. L. 1996, ApJ, 456, 393
Oliver, R. 2000, in Magnetic Fields and Solar Processes, ed. A. Wilson, ESA SP-448, 425

Oliver, R. 2001, in Magnetohydrodynamic Waves in Astrophysical Plasmas, ed. J. L. Ballester, \& B. Roberts, Universitat de les Illes Balears, in press

Pintér, B., Čadež, V. M., \& Roberts, B. 1999, A\&A, 346, 190

Roberts, B. 2000, Solar Phys., 193, 139

Schutgens, N. A. J. 1997, A\&A, 325, 352

Spiegel, E. A. 1957, ApJ, 126, 202

Stein, R. F., \& Spiegel, E. A. 1967, J. Acoust. Soc. Amer., 42, 866

Stix, M. 1970, A\&A, 4, 189

Tsubaki, T. 1988, in Solar and Stellar Coronal Structures and Dynamics, ed. R. C. Altrock, National Solar Observatory, 140, Fluid Dynam., 3, 89

Vršnak, B. 1993, Hvar Obs. Bull. 17, 23

Webb, A. R., \& Roberts, B. 1980, Solar Phys., 68, 71 